\title{
Meteor plasma trails: effects of external electric field
}

\author{
Y. S. Dimant ${ }^{1}$, M. M. Oppenheim ${ }^{1}$, and G. M. Milikh ${ }^{2}$ \\ ${ }^{1}$ Boston University, Boston, MA, USA \\ ${ }^{2}$ University of Maryland, College Park, MD, USA
}

Received: 8 July 2008 - Revised: 6 November 2008 - Accepted: 10 December 2008 - Published: 16 January 2009

\begin{abstract}
Meteoroids traversing the E-region ionosphere leave behind extended columns of elevated ionization known as the meteor plasma trails. To accurately interpret radar signals from trails and use them for diagnostics, one needs to model plasma processes associated with their structure and evolution. This paper describes a 3-D quantitative theory of the electrostatic interaction between a dense plasma trail, the ionosphere, and a DC electric field driven by an external dynamo. A simplified water-bag model of the meteor plasma shows that the highly conducting trail efficiently short-circuits the ionosphere and creates a vast region of currents that flow through and around the trail. We predict that the trail can induce electric fields reaching a few $\mathrm{V} / \mathrm{m}$, both perpendicular and parallel to the geomagnetic field. The former may drive plasma instabilities, while the latter may lead to strong heating of ionospheric electrons. We discuss physical and observational implications of these processes.
\end{abstract}

Keywords. Electromagnetics (Plasmas) - Ionosphere (Electric fields and currents; Ionospheric disturbances)

\section{Introduction}

Meteoroids entering the Earth's atmosphere with a hypersonic speed leave behind a trail of dense plasma visible to radars via specular and non-specular echoes (Baggaley and Webb, 1980; Levitskii et al., 1982; Jones and Jones, 1990; Elford and Elford, 2001; Galligan et al., 2004; Hocking, 2004), for review see Bronshten (1983); Ceplecha et al. (1998). Specular echoes are produced by reflection of HF or VHF waves from the large-scale electron density inhomogeneity associated with the plasma trail, largely when the radar line-of-sight is perpendicular to the trail axis (e.g.

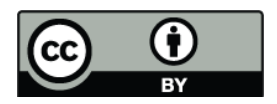

Correspondence to: Y. S. Dimant (dimant@bu.edu)
Kaiser and Closs, 1952; Lebedinec and Sosnova, 1968; Poulter and Baggaley, 1977; Jones and Jones, 1991). Nonspecular echoes result from Bragg scatter-off of smallerscale, magnetic-field-aligned plasma irregularities which develop as a result of plasma instabilities (Chapin and Kudeki, 1994a,b; Oppenheim et al., 2000; Dyrud et al., 2002, 2004). These plasma instabilities are driven by strong electric fields induced within or near the plasma trail.

The majority of meteoroids are too small to be visible even by sensitive optical equipment, so that radars often represent the only available diagnostic tool. To accurately interpret radar signals and use them for diagnostics, we need to understand the plasma processes associated with trail dynamics. In particular, we need to quantitatively describe the structure of macroscopic electric fields and currents associated with the trail drift and diffusion.

In Dimant and Oppenheim (2006a,b) we developed a 2-D theoretical description of meteor plasma trail diffusion and fields for arbitrarily dense trails that includes interactions between the trail and the background ionospheric plasma. In those papers, however, we disregarded external electric fields or neutral winds which are often present in the ionosphere. A spatially homogeneous and shearless neutral wind, perpendicular to the magnetic field, is equivalent to a uniform electric field in the neutral-wind frame of reference. The effect of this external electric field on the meteor trail was discussed by Chapin and Kudeki (1994a), Chapin (1996), and Oppenheim et al. (2000). However, these predominantly 2-D treatments missed the full 3-D polarization of the trail and effects associated with it.

In this paper, we develop a quantitative analytical 3-D theory of interaction between a dense meteor plasma trail and an external DC electric field, and discuss the physical and observational implications of this interaction. We summarize the results here.

A meteor trail distorts an otherwise uniform electric field due to the following. A dense plasma trail has a much

Published by Copernicus Publications on behalf of the European Geosciences Union. 


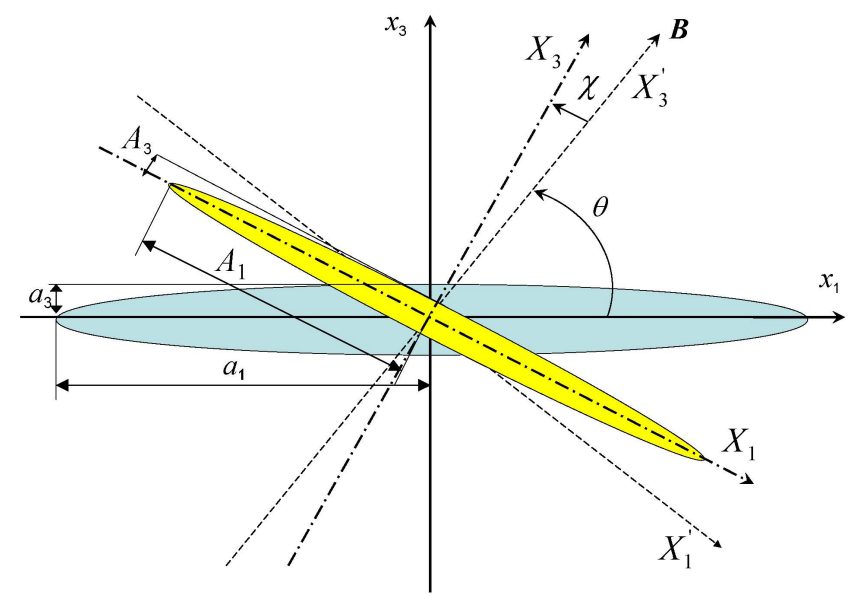

Fig. 1. Schematic of the elongated ellipsoidal trail and successive coordinate transformations $x_{i} \rightarrow X_{j}^{\prime} \rightarrow X_{k}$ (described in detail in Appendix A). Only the common magnetic field-trail axis plane is shown. The original trail is shaded blue; $\theta$ is the angle between $x_{1}$ and $\boldsymbol{B}$; axis $X_{3}^{\prime}$ is along $\boldsymbol{B}$. Rescaled along $\boldsymbol{B}$ by the small factor of $\epsilon$, Eq. (6), the trail is shaded yellow; $\chi$ is the angle between $X_{3}^{\prime}$ and $X_{3}$; the final axes $X_{k}$ are aligned with the principal axes of the rescaled ellipsoid; $a_{k}$ and $A_{k}$ are the semi-axes of the original and rescaled ellipsoids, respectively.

higher conductivity than the ambient E-region ionosphere, especially at night time. The component of a strong external DC electric field along the trail axis induces a strong current within the trail that cannot be closed in the ionosphere and must stop at the trail edges. As a result, the trail edges acquire net electric charges that partially cancel the external electric field within the trail. At the same time, far from the trail, the external electric field remains nearly undisturbed, creating a large potential difference projected onto the trail length. This leads to the formation of a transitional zone between the far ionosphere and the nearly equipotential meteor trail. This process looks similar to short-circuiting of a capacitor by a high-conductance wire. Unlike a capacitor, however, the ionospheric charge is distributed over a largevolume, magnetized, quasi-neutral plasma with the voltage sustained by the ionospheric dynamo (Kelley, 1989). In the most typical cases, the external DC electric field is not fully expelled from the trail.

The transitional zone between the two different plasma density distributions contains spatially inhomogeneous electric fields which increase dramatically near the meteor trail edges. These induced fields may drive plasma instabilities which give rise to plasma density irregularities responsible for non-specular radar echoes. Additionally, the field induced near the trail edges can have a significant component along the magnetic field. This field can energize electrons dramatically.

Polarization electric fields near the trail edges give rise to electric currents which flow from the ionosphere into the trail on one side of the trail and flow out into the ionosphere from the other side. This effect results in a net trail current which complements the current loop associated with the trail diffusion (Dimant and Oppenheim, 2006a). Unlike the induced electric fields that decrease as the trail diffuses, the polarization trail current stays roughly the same while the trail density remains sufficiently large. Near the trail, the currents flowing in and out of trail may strongly heat the plasma (mainly electrons), leading to a number of observable features, including airglow. Also, strong plasma heating could affect the rate of diffusion and may manifest itself in some other unexpected ways.

The paper is organized as follows. In Sect. 2, we formulate the problem and give some description of model. In Sect. 3, we find the general solution of the potential around a highly conductive 3-D ellipsoidal trail immersed in a homogeneous ionospheric plasma with an external DC electric field. In Sect. 4, we find ionospheric currents flowing both through the trail surface and within the trail, determine the internal trail electric field, and close the entire solution. In Sect. 5, we estimate the electron heating in and near the trail. In Sect. 6, we briefly discuss our findings and make some predictions regarding potentially observable physical effects caused by the process under study. Section 7 gives concluding remarks. In Appendices A and B, we present some mathematical details.

\section{Description of model}

In this section, we describe a simplified model of a meteor plasma trail and the surrounding medium that will enable us to catch the major features of how the external DC electric field polarizes the meteor plasma trail, resulting in distortions and amplifications of the field in the near-trail ionosphere. To this end, we assume a spatially homogeneous ionosphere with a dense plasma trail and an external electric field with a significant component along the trail. If the trail conductivity were infinitely high then the polarized trail would entirely expel the electric field. For the majority of real meteor trails, however, this ideal case is not a valid approximation. We consider then the more general and realistic case of finite trail conductivity, when the trail still contains a significant electric field.

Meteor plasma trails often extend over many kilometers in length, while their diameter is several orders of magnitude smaller. In the course of relatively slow trail diffusion, the trail length remains nearly constant, while its transverse sizes vary from several meters to tens of meters or even more until the dense trail effectively disappears. The diffusion coefficient is inversely proportional to the neutral atmosphere density that decreases with increasing height, approximately following the barometric formula. Due to this, the trail should have a trumpet-like shape with a larger trail diameter at higher altitudes. However, to make an analytic 
solution possible, we assume cigar-like 3-D ellipsoidal shape of the trail described below by Eq. (3).

We choose the following coordinate system: the $\boldsymbol{x}_{1}$-axis is directed along the main trail axis, the $\boldsymbol{x}_{2}$-axis is directed perpendicular to both the trail axis and $\boldsymbol{B}$, the $\boldsymbol{x}_{3}$-axis is perpendicular to the trail axis and lies in the common $\boldsymbol{B}$-trail-axis plane, where $\theta$ is the angle between $\boldsymbol{B}$ and $\boldsymbol{x}_{1}$, see Fig. 1 . The external uniform DC electric field, $\boldsymbol{E}^{(0)}=\left(E_{1}^{(0)}, E_{2}^{(0)}, E_{3}^{(0)}\right)$, is perpendicular to $\boldsymbol{B}$. We will show that the most important component of $\boldsymbol{E}^{(0)}$ lies in the $x_{1} x_{3}$-plane.

To describe the formation of the total electrostatic field, $\boldsymbol{E}=-\nabla \Phi$, where the electrostatic potential $\Phi$ includes both the external DC field and the polarization field caused by the highly conducting trail, we will use electron and ion fluid equations combined with the quasineutrality condition. The isothermal fluid equations for the diffusion regime include two inertialess momentum equations and two continuity equations for electrons and ions. The quasineutrality condition makes the Poisson equation for the electric potential unnecessary. This set of equations can be readily reduced to two coupled nonlinear partial differential equations for the common plasma density, $n$, and $\Phi$ :

$$
\begin{array}{r}
\partial_{t} n+\nabla \cdot \boldsymbol{\Gamma}_{i}=0, \\
\nabla \cdot \boldsymbol{j}=e \nabla \cdot\left(\boldsymbol{\Gamma}_{i}-\boldsymbol{\Gamma}_{e}\right)=0
\end{array}
$$

where $\boldsymbol{j}$ is the total current density and the diffusion flux densities, $\boldsymbol{\Gamma}_{e, i}$, are given by

$$
\begin{aligned}
\boldsymbol{\Gamma}_{i} & \approx-n \frac{\nabla\left(e \Phi+T_{i} \ln \left(n / n_{0}\right)\right)}{m_{i} v_{i n}}, \\
\boldsymbol{\Gamma}_{e \|} & \approx n \frac{\nabla_{\|}\left(e \Phi-T_{e} \ln \left(n / n_{0}\right)\right)}{m_{e} v_{e n}}, \\
\boldsymbol{\Gamma}_{e \perp} & \approx n\left[\frac{v_{e n} \nabla_{\perp}\left(e \Phi-T_{e} \ln \left(n / n_{0}\right)\right)}{m_{e} \Omega_{e}^{2}}\right. \\
& \left.+\frac{\hat{b} \times \nabla_{\perp}\left(e \Phi-T_{e} \ln \left(n / n_{0}\right)\right)}{m_{e} \Omega_{e}}\right] .
\end{aligned}
$$

Here $n_{0}$ is the background ionospheric density; $T_{e, i}$ are the electron and ion temperatures, $m_{e, i}$ are the electron and ion masses; $\Omega_{e, i}=e B / m_{e, i}$ are the electron and ion gyrofrequencies, $v_{e n} \ll \Omega_{e}$ and $v_{i n} \gg \Omega_{i}$ are the electron-neutral and ionneutral collision frequencies, respectively; $e$ is the elementary charge; the subscripts $\|$ and $\perp$ pertain to the directions parallel and perpendicular to the magnetic field $\boldsymbol{B}$, respectively; $\hat{b}$ is the unit vector along $\boldsymbol{B}$. The first term in the right-hand side (RHS) of Eq. (2c) describes the electron Pedersen flux, while the second term describes the electron Hall flux. In more general adiabatic processes, we would have additional factors $\gamma_{e, i}$ in front of $T_{e . i} \ln \left(n / n_{0}\right)$. In obtaining Eq. (2), we have used the fact that the effects discussed in this paper are most pronounced at altitudes between 90 and $120 \mathrm{~km}$, where electrons are magnetized, while ions are unmagnetized due to frequent collisions with the neutral atmosphere.
In this paper, for simplicity, we will use a water-bag model of the meteor plasma trail, which assumes a given uniform plasma density, $n^{\text {int }}$, within a volume restricted by a given boundary surface (e.g. Hysell and Drexler, 2006). We will also neglect disturbances of the background plasma density (Oppenheim and Dimant, 2006), assuming that beyond the trail $n \approx n_{0}$. To make the analytical solution possible, we assume a 3-D ellipsoidal, i.e., cigar-like shape of the plasma trail,

$\frac{x_{1}^{2}}{a_{1}^{2}}+\frac{x_{2}^{2}}{a_{2}^{2}}+\frac{x_{3}^{2}}{a_{3}^{2}}=1$.

We assume that the ellipsoid is extended along the $x_{1}$-axis,

$a_{1} \gg a_{3} \sim(1+\psi)^{1 / 2} a_{2}$,

and this direction is not too close to that of the magnetic field,

$\sin \theta \gg \epsilon, \frac{a_{3}}{a_{1}}$,

where we introduced useful dimensionless parameters

$\psi=\frac{v_{e n} v_{i n}}{\Omega_{e} \Omega_{i}}, \quad \Theta_{0}=\left(\frac{m_{e} v_{e n}}{m_{i} \nu_{i n}}\right)^{1 / 2} \approx 1.35 \times 10^{-2}$,

and

$\epsilon=\Theta_{0}(1+\psi)^{1 / 2} \ll 1$,

The widely used parameter $\psi$ represents the ratio between the Pedersen mobility of magnetized electrons and that of largely unmagnetized ions, while $\Theta_{0}^{2}$ is the ratio between the ion and electron mobilities along the magnetic field. Across the E-region altitudes, the values of the collision frequencies are such (e.g. Kelley, 1989; Schunk and Nagy, 2000) that their ratio is approximately constant, $v_{e n} / v_{i n} \simeq 10$ (Dimant and Milikh, 2003; Dimant and Oppenheim, 2006a). This provides an approximate constancy of $\Theta_{0}$ along with its numerical value specified in Eq. (5). At the same time, the parameter $\psi$ decreases exponentially with increasing altitude, reaching rather small values at the top altitudes of meteor trail formation (for a model of altitude dependence of $\psi$, see Dimant and Oppenheim, 2004, Fig. 2). The smallness of $\epsilon$ for any $\psi$ within this range follows from both $\Theta_{0} \ll 1$ and $\Theta_{0} \sqrt{\psi}=v_{e n} / \Omega_{e} \ll 1$. Note that the aforementioned specific models of $\psi$ and $\Theta_{0}$ pertain to the undisturbed E-region ionosphere. At the same time, this paper shows that a strong induced electric field near the meteor trail can result in significant electron heating and the corresponding local modifications of $v_{e n}$. We discuss this effect and its implications in Sects. 5 and 6.

To apply Eq. (1b) to the two uniform plasmas separated by the ellipsoidal interface, we should make an anisotropic coordinate transformation from the original, $x_{i}$, to new coordinates, $X_{k}$. The entire transformation consists of a number of sequential steps, described in detail in Appendix A. 


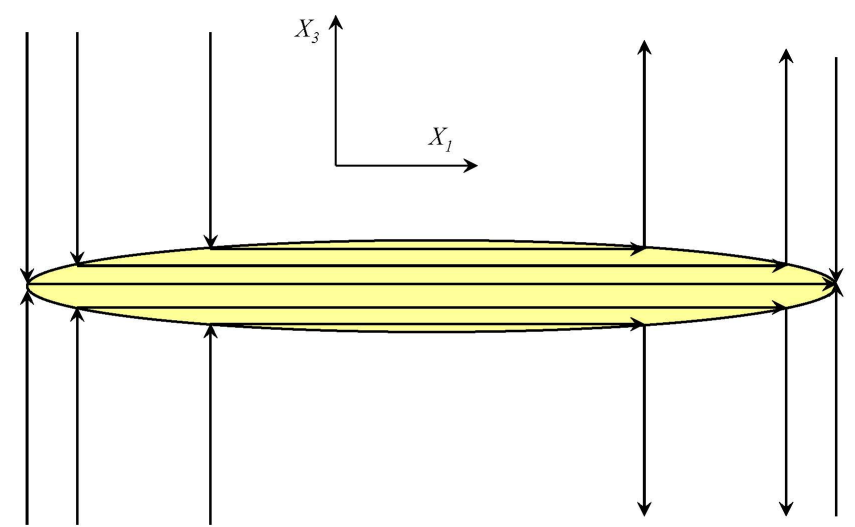

Fig. 2. Schematic diagram of current closure through the ellipsoidal trail (light-yellow shading). Vertical arrows depict ionospheric currents directed mainly along $X_{3}$, while horizontal arrows depict trail currents which flow along $X_{1}$.

First, we rotate the coordinate system in order to make one of its axes parallel to the magnetic field. Second, to adjust for strongly different conductivities along and across the magnetic field, we make an anisotropic scaling transformation. In the new coordinates, the equation $\nabla \cdot \boldsymbol{j}=0$ reduces to Laplace's equation for the electrostatic potential, but the 3-D ellipsoid, Eq. (3), loses its simple canonical form. In order to restore this form, we make a final small coordinate rotation. All these transformations are performed in the $x_{1} x_{3}$-plane. When the trail axis is perpendicular to $\boldsymbol{B}\left(\theta=90^{\circ}\right)$ only the scaling is necessary.

The entire transformations from $x_{i}$ to $X_{k}$ and back are described by Eqs. (A9) and (A14). To the zeroth-order accuracy with respect to small parameters $\epsilon$ and $a_{3} / a_{1}$, see Eqs. (6) and (4), we have

$x_{1} \approx \frac{X_{1}}{\sin \theta}+\frac{X_{3} \cos \theta}{\epsilon}$,

$x_{2}=X_{2}$,

$x_{3} \approx \frac{X_{3} \sin \theta}{\epsilon}$,

and, vice versa,

$X_{1} \approx x_{1} \sin \theta-x_{3} \cos \theta$,

$X_{2}=x_{2}$,

$X_{3} \approx \frac{\epsilon x_{3}}{\sin \theta}$.

The covariant electric field transforms according to $E_{i}=\sum_{k=1}^{3}\left(\partial X_{k} / \partial x_{i}\right) E_{X_{k}}$, see Eqs. (A12), (A11), and (A13), while the current density transforms according to $j_{i}=\operatorname{det}\left[\partial X_{k} / \partial x_{i}\right] \sum_{k=1}^{3}\left(\partial x_{i} / \partial X_{k}\right) J_{X_{k}}$, where $\operatorname{det}\left[\partial X_{k} / \partial x_{i}\right]=n / N=\epsilon$ and $N$ is the plasma density in the renormalized coordinates $X_{k}$. In these coordinates, the current density is given by Eq. (A15).
In the renormalized coordinates $X_{k}$, the electrostatic potential obeys isotropic Laplace's equation

$\sum_{i=1}^{3} \frac{\partial^{2} \Phi}{\partial X_{i}^{2}}=0$

while the coordinates are the principal axes of the rescaled ellipsoid, Eq. (3),

$\frac{X_{1}^{2}}{A_{1}^{2}}+\frac{X_{2}^{2}}{A_{2}^{2}}+\frac{X_{3}^{2}}{A_{3}^{2}}=1$,

where, to the zeroth-order accuracy,

$A_{1} \approx a_{1} \sin \theta, \quad A_{2}=a_{2}, \quad A_{3} \approx \frac{\epsilon a_{3}}{\sin \theta}$.

According to Eq. (4), the new semi-axes differ significantly,

$A_{1} \gg A_{2} \gg A_{3}$.

We will use this fact in Sect. 3 and Appendix B in order to approximate complicated general analytical expressions.

Equation (9) is applicable to both the dense plasma inside the ellipsoidal trail and to the tenuous background plasma beyond it. The boundary conditions on the interface separating the two plasmas are: (1) continuity of the electrostatic potential (resulting in continuity of the tangential electric field) and (2) continuity of the total current density normal to the interface. The second boundary condition implies that there are no surface currents, though the boundary surface can accumulate significant surface charges that result in trail polarization and discontinuity of the normal electric field. There are no surface currents because straight current lines within the trail can only cross the ellipsoidal interface, but they never flow strictly within it, as illustrated by Fig. 2. The current lines within the trail are straight because the electric field there is uniform - the fact which we initially postulate and then prove through a self-consistent solution.

In the background plasma sufficiently far from the trail, the total electric field is dominated by the external electric field, $\boldsymbol{E}^{(0)}$. This field is perpendicular to $\boldsymbol{B}$ in all coordinate systems, so that in the $X_{i}$-coordinates it lies mainly in $X_{1} X_{2}$ plane, and we will neglect its possible small $X_{3}$-component. The external field represents an asymptotic boundary condition for Eq. (9) at sufficiently large distances from the trail.

Within the meteor plasma trail, where we denote all quantities by the superscript "int", we assume that the trail contains an internal uniform electric field, $\boldsymbol{E}^{\text {int }}$. This field will remain unknown until we solve for the electric potential beyond the trail and close the entire solution in Sect. 4. Later we will show that the assumption of the uniform internal field is automatically consistent with our original assumptions of the water-bag model and the ellipsoidal interface. The uniform internal field corresponds to a potential with the linear coordinate dependence,

$\Phi^{\mathrm{int}}=-\sum_{i=1}^{3} E_{X_{i}}^{\mathrm{int}} X_{i}$ 
which is evidently a solution of Laplace's Eq. (9). We will use this fact in the next section.

\section{Electric field beyond trail}

In this section we determine the electric field in the regions outside the trail. In Sect. 3.1 we obtain the general analytical solution for the potential, while in Sect. 3.2 we determine the electric field on the ellipsoidal interface that separates the dense plasma trail from the tenuous ionosphere.

\subsection{General solution of potential}

Here we obtain the general analytical solution of Laplace's Eq. (9) in the background plasma beyond the ellipsoidal trail. On the ellipsoidal interface defined by Eq. (10), the electrostatic potential $\Phi$ equals the linear potential of the trail given by Eq. (13), while at large distances from the boundary ellipsoid we have $\Phi \approx \Phi^{(0)}\left(X_{i}\right)$, where $\Phi^{(0)}\left(X_{i}\right)$ corresponds to the external electric field, $\boldsymbol{E}^{(0)}$,

$\Phi^{(0)}=-\sum_{i=1}^{3} E_{X_{i}}^{(0)} X_{i}$

Solving Eq. (9) becomes much easier if we extrapolate $\Phi^{\text {int }}$ from Eq. (13) to the entire space and introduce the difference

$\Delta \Phi=\Phi-\Phi^{\mathrm{int}}$

which should be a solution of Laplace's Eq. (9) because both $\Phi$ and $\Phi^{\text {int }}\left(X_{i}\right)$ are. For $\Delta \Phi$, the original boundary problem reduces to the problem of an equipotential ellipsoid in an external field $\Delta E_{X_{i}}^{(0)} \equiv E_{X_{i}}^{(0)}-E_{X_{i}}^{\text {int }}$. This problem is equivalent to the well-known vacuum problem of an uncharged conducting ellipsoid in a uniform electric field (e.g. Landau and Lifshitz, 1960, Sect. 4). The unique potential that satisfies Laplace's equation with the boundary conditions $\Delta \Phi=0$ on the ellipsoid surface and $\Delta \Phi \rightarrow-\sum_{i=1}^{3} \Delta E_{X_{i}}^{(0)} X_{i}$ as $\left|X_{i}\right| \rightarrow \infty$, can be represented as a linear superposition of partial potentials,

$\Delta \Phi=\sum_{i=1}^{3} \Delta \Phi^{(i)}$

where each partial potential $\Delta \Phi^{(i)}=0$ on the ellipsoid surface and $\Delta \Phi^{(i)} \rightarrow-\Delta E_{X_{i}}^{(0)} X_{i}$ as $\left|X_{i}\right| \rightarrow \infty$.

The conventional way of solving Laplace's equation with an ellipsoidal boundary is via the use of ellipsoidal coordinates confocal with the ellipsoidal boundary (Landau and Lifshitz, 1960). The three ellipsoidal coordinates are defined as the three independent real roots of the cubic equation,

$\frac{X_{1}^{2}}{A_{1}^{2}+u}+\frac{X_{2}^{2}}{A_{2}^{2}+u}+\frac{X_{3}^{2}}{A_{3}^{2}+u}=1$, for the unknown $u$. Since Eq. (12) requires $A_{1}>A_{2}>A_{3}$, we order the three roots, $u=\xi, \eta, \zeta$, according to constraints

$\xi \geq-A_{3}^{2}, \quad-A_{2}^{2} \leq \eta \leq-A_{3}^{2}, \quad-A_{1}^{2} \leq \zeta \leq-A_{2}^{2}$

The coordinates $\eta$ and $\zeta$ are always negative, while $\xi$ is negative inside, positive outside, and equals zero on the ellipsoidal interface described by Eq. (10). The mutually orthogonal surfaces of constant $\xi, \eta$, and $\zeta$ are ellipsoids and hyperboloids of one and two sheets, respectively. In terms of the ellipsoidal coordinates, the renormalized Cartesian coordinates $X_{i}$ are given by

$$
\begin{aligned}
& X_{1}= \pm \sqrt{\frac{\left(A_{1}^{2}+\xi\right)\left(A_{1}^{2}+\eta\right)\left(A_{1}^{2}+\zeta\right)}{\left(A_{1}^{2}-A_{3}^{2}\right)\left(A_{1}^{2}-A_{2}^{2}\right)}}, \\
& X_{2}= \pm \sqrt{\frac{\left(A_{2}^{2}+\xi\right)\left(A_{2}^{2}+\eta\right)\left(A_{2}^{2}+\zeta\right)}{\left(A_{1}^{2}-A_{2}^{2}\right)\left(A_{3}^{2}-A_{2}^{2}\right)}}, \\
& X_{3}= \pm \sqrt{\frac{\left(A_{3}^{2}+\xi\right)\left(A_{3}^{2}+\eta\right)\left(A_{3}^{2}+\zeta\right)}{\left(A_{1}^{2}-A_{3}^{2}\right)\left(A_{2}^{2}-A_{3}^{2}\right)}} .
\end{aligned}
$$

Beyond the ellipsoid diametral planes, $X_{i}=0$, each set of $\xi, \eta, \zeta$, yields eight symmetric points corresponding to different signs of $X_{i}$.

The element of length in the ellipsoidal coordinates is determined by $d l^{2}=h_{\xi}^{2} d \xi^{2}+h_{\eta}^{2} d \eta^{2}+h_{\zeta}^{2} d \zeta^{2}$, where

$$
\begin{aligned}
& h_{\xi}=\frac{\sqrt{(\xi-\eta)(\xi-\zeta)}}{2 R_{\xi}}, \\
& h_{\eta}=\frac{\sqrt{(\eta-\zeta)(\xi-\eta)}}{2 R_{\eta}}, \\
& h_{\zeta}=\frac{\sqrt{(\xi-\zeta)(\zeta-\eta)}}{2 R_{\zeta}},
\end{aligned}
$$

and (for $u=\xi, \eta, \zeta$ )

$R_{u}=\sqrt{\left(u+A_{1}^{2}\right)\left(u+A_{2}^{2}\right)\left(u+A_{3}^{2}\right)}$.

In the ellipsoidal coordinates, $\xi, \eta, \zeta$, the boundary conditions for $\Delta \Phi^{(i)}$ become

$$
\left.\Delta \Phi^{(i)}\right|_{\xi=0}=0,\left.\quad \Delta \Phi^{(i)}\right|_{\xi \rightarrow \infty} \rightarrow-\Delta E_{X_{i}}^{(0)} X_{i} .
$$

Rewriting Eq. (9) in the ellipsoidal coordinates and multiplying the result by $(\xi-\eta)(\eta-\zeta)(\zeta-\xi) / 4$, we obtain

$$
\begin{gathered}
(\eta-\zeta) R_{\xi} \frac{\partial}{\partial \xi}\left(R_{\xi} \frac{\partial \Delta \Phi^{(i)}}{\partial \xi}\right) \\
+(\zeta-\xi) R_{\eta} \frac{\partial}{\partial \eta}\left(R_{\eta} \frac{\partial \Delta \Phi^{(i)}}{\partial \eta}\right) \\
+(\xi-\eta) R_{\zeta} \frac{\partial}{\partial \zeta}\left(R_{\zeta} \frac{\partial \Delta \Phi^{(i)}}{\partial \zeta}\right)=0 .
\end{gathered}
$$




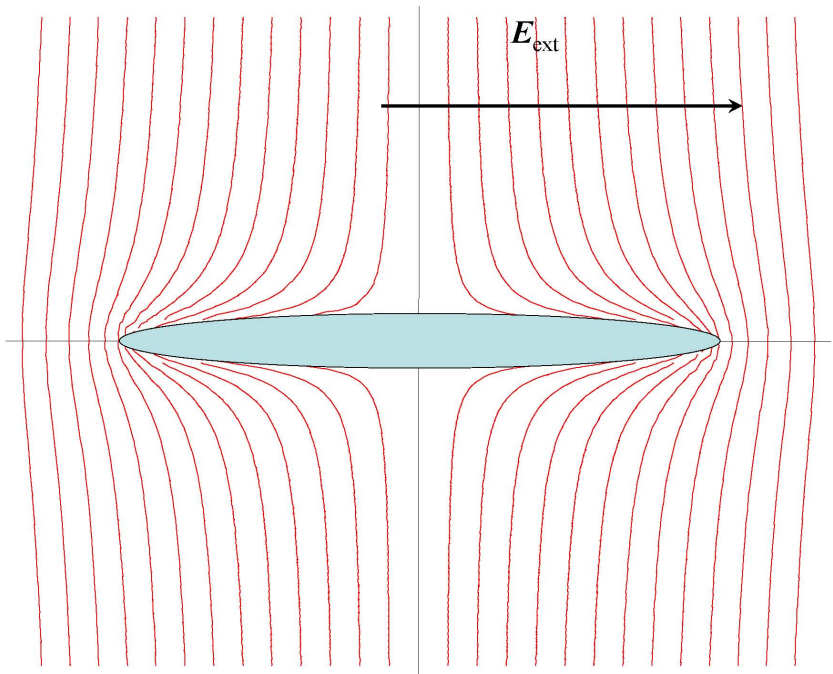

Fig. 3. Contours of equal potential (red curves) around an infinitely conducting ellipsoidal trail (shaded light blue). The external electric field direction (shown by the black arrow) is parallel to the major ellipsoid axis. The equipotential contours flow around the equipotential trail, resulting in build-up of strong electric fields near its boundary.

We will seek the solution of the boundary problem for $\Delta \Phi^{(i)}$ in the form

$\Delta \Phi^{(i)}=-\Delta E_{X_{i}}^{(0)} X_{i} G_{X_{i}}(\xi)$,

where the dimensionless functions $G_{X_{i}}(\xi)$ satisfy

$G_{X_{i}}(\xi) \rightarrow\left\{\begin{array}{l}0 \text { at } \xi \rightarrow 0 \\ 1 \text { at } \xi \rightarrow \infty\end{array}\right.$

Now we make use of the fact that the potential $-\Delta E_{X_{i}}^{(0)} X_{i}$, linearly dependent on $X_{i}$, is itself a solution of Laplace's equation. This allows us to eliminate all derivatives with respect to $\eta$ and $\zeta$ in Eq. (23), making it an ordinary differential equation for $G_{X_{i}}$,

$\frac{d^{2} G_{X_{i}}}{d \xi^{2}}+\frac{d G_{X_{i}}}{d \xi} \frac{d}{d \xi} \ln \left[R_{\xi}\left(A_{i}^{2}+\xi\right)\right]=0$.

Solving this equation with the asymptotic boundary conditions given by Eq. (25), we obtain

$G_{X_{i}}(\xi)=\int_{0}^{\xi} \frac{d \xi}{\left(A_{i}^{2}+\xi\right) R_{\xi}} / \int_{0}^{\infty} \frac{d \xi}{\left(A_{i}^{2}+\xi\right) R_{\xi}}$

Recalling Eqs. (15) and (16), we obtain the total potential

$\Phi=\sum_{i=1}^{3}\left(-E_{X_{i}}^{\mathrm{int}} X_{i}+\Delta \Phi^{(i)}\right)$

where

$\Delta \Phi^{(i)}(\xi, \eta, \zeta)=\frac{\left(E_{X_{i}}^{\mathrm{int}}-E_{X_{i}}^{(0)}\right) X_{i} A_{i}^{3}}{2 \Lambda_{i}} \int_{0}^{\xi} \frac{d \xi}{\left(A_{i}^{2}+\xi\right) R_{\xi}}$
$\Lambda_{i}=\frac{A_{i}^{3}}{2} \int_{0}^{\infty} \frac{d \xi}{\left(A_{i}^{2}+\xi\right) R_{\xi}}$.

The integrals in Eq. (27) can be reduced to canonical elliptic integrals, as described in Appendix B. We have chosen the normalization in such a way that the constants $\Lambda_{i}$ become dimensionless and allow simple approximations given below by Eq. (32).

\subsection{Electric field on boundary surface}

Equation (26) describes the spatial distribution of the total electric potential in the entire space beyond the trail. Figure 3 illustrates the solution for the extreme case of an infinitely conducting and hence equipotential trail. This figure shows that the surfaces of equal potential flow around the trail and thicken dramatically near its edges. This means that a significant electric field builds up near the ionospheretrail interface. In the more general case of a finite conductivity trail, the electric field will partially penetrate into the trail. Of principal interest for us is the electric field directly on the interface defined by Eq. (10), especially its induced part, $\Delta E_{X_{j}}^{(i)}=-\partial \Delta \Phi^{(i)} / \partial X_{j}$ (here and below the superscripts $(i)$ denote the partial potentials and fields, while the subscripts $(j)$ denote different vector components). This largest-amplitude field determines the ionospheric currents that flow into or out of the trail. The electric field $\Delta E_{X_{j}}^{(i)}$ is the solution for the boundary condition of the equipotential trail, so that on the ellipsoidal interface this field must be normal to it. It is easy to verify that the components of a unit vector which is normal to an ellipsoidal surface of constant $\xi$ are given by $\hat{n}_{j}=X_{j} /\left(A_{j}^{2} \sqrt{P}\right)$, where

$P=\frac{X_{1}^{2}}{A_{1}^{4}}+\frac{X_{2}^{2}}{A_{2}^{4}}+\frac{X_{3}^{2}}{A_{3}^{4}}$,

so that the vector components of the normal electric field are

$\Delta E_{X_{j}}^{(i)}=\frac{X_{j} \Delta E^{(i)}}{A_{j}^{2} \sqrt{P}}$.

Here $\quad \Delta E^{(i)}=-d \Delta \Phi^{(i)} / d n=-\left(1 / h_{\xi}\right) \partial \Delta \Phi^{(i)} / \partial \xi \quad$ is the partial field amplitude and $h_{\xi}$ is defined by Eq. (20a). On the ellipsoidal interface $\xi=0$, we have $d \Delta \Phi^{(i)} /\left.d n\right|_{\xi=0}=\left.\left(2 A_{1} A_{2} A_{3} / \sqrt{\eta \zeta}\right) \partial \Delta \Phi^{(i)}\right|_{\xi=0} / \partial \xi$. Manipulating with Eq. (19), we obtain $\left.\eta \zeta\right|_{\xi=0}=A_{1}^{2} A_{2}^{2} A_{3}^{2} P$, where $X_{i}$ are related by Eq. (10), so that $\Delta E^{(i)}=-(2 / \sqrt{P}) \partial \Delta \Phi^{(i)} /\left.\partial \xi\right|_{\xi=0}$. From Eq. (27a), we obtain $\partial \Delta \Phi^{(i)} /\left.\partial \xi\right|_{\xi=0}=\left.A_{i} \Delta \Phi^{(i)}\right|_{\xi=0} / 2 A_{1} A_{2} A_{3} \Lambda_{i}$, so that

$\Delta E^{(i)}=\frac{\left(E_{X_{i}}^{(0)}-E_{X_{i}}^{\mathrm{int}}\right) X_{i} A_{i}}{A_{1} A_{2} A_{3} \Lambda_{i} \sqrt{P}}$. 
Using Eqs. (26) and (29), we obtain the total vector field on the boundary surface,

$$
\left.E_{X_{j}}\right|_{\xi=0}=E_{X_{j}}^{\mathrm{int}}+\left.\frac{X_{j}}{A_{j}^{2} P} \sum_{k=1}^{3} \frac{\left(E_{X_{k}}^{(0)}-E_{X_{k}}^{\mathrm{int}}\right) X_{k} A_{k}}{A_{1} A_{2} A_{3} \Lambda_{k}}\right|_{\xi=0} .
$$

Now we simplify these general results using strong inequalities given by Eq. (12). Assuming that $A_{2} / A_{1}$ and $A_{3} / A_{2}$ are small, according to Eq. (B7) from Appendix B, the integral constants $\Lambda_{i}$ in Eq. (27b) can be approximated by

$\Lambda_{1} \approx \ln \left(\frac{4 A_{1}}{A_{2}}\right)-1$,

$\Lambda_{2} \approx \frac{A_{2}}{A_{1}}, \quad \Lambda_{3} \approx \frac{A_{3}^{2}}{A_{1} A_{2}}$.

Furthermore, where

$\frac{A_{3}^{2}}{A_{1}} \ll \frac{A_{3}^{2}}{A_{2}} \ll\left|X_{3}\right| \leq A_{3}$,

the two first terms in the RHS of Eq. (28) are small compared to the last one,

$P \approx \frac{X_{3}^{2}}{A_{3}^{4}}=\frac{1}{A_{3}^{2}}\left(1-\frac{X_{1}^{2}}{A_{1}^{2}}-\frac{X_{2}^{2}}{A_{2}^{2}}\right)$.

Equation (34) is invalid only on a narrow belt area around the edge ellipse that lies in the plane, $X_{3}=0$,

$\left|X_{3}\right| \lesssim \frac{A_{3}^{2}}{A_{2}} \ll A_{3}$

whose contribution to the entire current balance is negligible. The dimensionless parameters $\Lambda_{i}$, given by Eq. (27b) or approximately by Eq. (32), depend on the ratios of the ellipsoid semi-axes $A_{i}$. For $\left.\left.\Delta E_{X_{j}}\right|_{\xi=0} \equiv E_{X_{j}}\right|_{\xi=0}-\left.E_{X_{j}}^{\text {int }}\right|_{\xi=0}$, on most of the boundary surface, we obtain

$$
\begin{aligned}
& \left.\left.\Delta E_{X_{1}}\right|_{\xi=0} \approx \frac{A_{3}^{2} X_{1}}{A_{1}^{2} X_{3}}\left|\Delta E_{X}\right|_{\xi=0} \ll \Delta E_{X_{2}}\right|_{\xi=0}, \\
& \left.\left.\Delta E_{X_{2}}\right|_{\xi=0} \approx \frac{A_{3}^{2} X_{2}}{A_{2}^{2} X_{3}}\left|\Delta E_{X}\right|_{\xi=0} \ll \Delta E_{X_{3}}\right|_{\xi=0}, \\
& \left.\Delta E_{X_{3}}\right|_{\xi=0} \approx\left|\Delta E_{X}\right|_{\xi=0 .} .
\end{aligned}
$$

Here, in accord with Eqs. (30), (32), and (34), the amplitude of the electric field normal to the interface is given by

$$
\begin{aligned}
\left|\Delta E_{\boldsymbol{X}}\right|_{\xi=0} & =\sum_{i=1}^{3}\left|\Delta E_{\boldsymbol{X}}^{(i)}\right|_{\xi=0} \\
& \approx \frac{A_{3} X_{1} \Delta E_{X_{1}}^{(0)}}{A_{2} X_{3} \Lambda_{1}}+\frac{A_{3} X_{2} \Delta E_{X_{2}}^{(0)}}{A_{2} X_{3}}+\Delta E_{X_{3}}^{(0)},
\end{aligned}
$$

where $\Delta E_{X_{i}}^{(0)} \equiv E_{X_{i}}^{(0)}-E_{X_{i}}^{\text {int }}$. According to Eq. (36), beyond the narrow belt area described by Eq. (35), the normal electric field is directed mainly along $X_{3}$ because the equipotential ellipsoid in the $X_{i}$ coordinates is flattened in this direction. The contributions to $\left|\Delta E_{X}\right|_{\xi=0}$ from the components of the external electric field in the directions perpendicular to the major trail axis, $\Delta E_{X_{2,3}}^{(0)}$, are of the order of these components at most. The contribution from the electric field component along the trail axis, $\Delta E_{1}^{(0)}$, is, however, much more significant. Unless the coordinate $X_{1}$ is too close to the plane $X_{1}=0, X_{1} \lesssim A_{2} \Lambda_{1} \ll A_{1}$, the first term in the RHS of Eq. (37) is $\sim A_{1} / A_{2} \gg 1$ times larger than the two remaining terms. Neglecting contributions from other components of $\Delta \boldsymbol{E}^{(0)}$ and using Eq. (36c), we obtain on most of the trail surface $\left.\Delta \boldsymbol{E}_{\boldsymbol{X}}\right|_{\xi=0} \approx\left|\Delta E_{\boldsymbol{X}}\right|_{\xi=0} \hat{X}_{3}$, where $\hat{X}_{3}$ is the unit vector along $X_{3}$ and

$\left|\Delta E_{X}\right|_{\xi=0} \approx \frac{A_{3} X_{1} \Delta E_{X_{1}}^{(0)}}{A_{2} X_{3} \Lambda_{1}}$.

The effect of field amplification increases when approaching the plane $X_{3}=0$, and saturates on the narrow belt described by Eq. (35), where one should use for $P$ and $\Delta E_{j}$ general Eqs. (28-30). The amplification factor is, to within an order of magnitude, $\left|\Delta E_{X}\right|_{\xi=0} / \Delta E_{X_{1}}^{(0)} \sim X_{1} / A_{3} \Lambda_{1}$. According to Eq. (32), for $A_{1} / A_{3} \lesssim 1000, \Lambda_{1}$ reaches $\sim 7$, so that well beyond the plane $X_{1}=0$, and especially when approaching the ends, $X_{1}= \pm A_{1}$, the amplification factor can reach a few hundred. Note, however, that in the narrow belt area around the plane $X_{3}=0$, the normal electric field is no longer nearly parallel to the $X_{3}$-axis but, depending on the specific location, can have any direction. Note also that we disregard here the effect of field-induced electron heating on ionospheric parameters near the trail (see the discussion in the end of Sect. 5).

In the original coordinates $x_{j}$, the boundary electric field, $\left.\Delta E_{i}\right|_{\xi=0}$, could be obtained by using Eq. (A12). More convenient, however, is to use the following approach. The field $\Delta \boldsymbol{E}=-\nabla \sum_{i=1}^{3} \Delta \Phi^{(i)}$ is a covariant vector, so that its components in the original coordinates, $\left.\Delta E_{j}\right|_{\xi=0}$, are given by

$\left.\Delta E_{j}\right|_{\xi=0}=\left.\sum_{k=1}^{3} \frac{\partial X_{k}}{\partial x_{j}} \Delta E_{X_{k}}\right|_{\xi=0}$.

According to Eq. (29), we have

$\left.\Delta E_{X_{k}}\right|_{\xi=0}=\frac{X_{k}\left|\Delta E_{X}\right|_{\xi=0}}{A_{k}^{2} \sqrt{P}}=\frac{1}{2} \frac{\partial S}{\partial X_{k}} \frac{\left|\Delta E_{X}\right|_{\xi=0}}{\sqrt{P}}$,

where we introduced $S \equiv \sum_{k=1}^{3} X_{k}^{2} / A_{k}^{2}$. According to Eqs. (3) and (10), on the ellipsoidal interface we have $\left.S\right|_{\xi=0}=1=\sum_{j=1}^{3} x_{j}^{2} / a_{j}^{2}$, so that we can consider $S$ as a scalar function of coordinates, which remains invariant with respect to coordinate transformations. As a result, we obtain from Eqs. (39) and (40)

$\left.\Delta E_{j}\right|_{\xi=0}=\frac{1}{2} \frac{\partial S}{\partial x_{j}} \frac{\left|\Delta E_{X}\right|_{\xi=0}}{\sqrt{P}}=\frac{x_{j}\left|\Delta E_{X}\right|_{\xi=0}}{a_{j}^{2} \sqrt{P}}$ 
where $P$ and $\left|\Delta E_{X}\right|_{\xi=0}$ are functions of the renormalized coordinates, $X_{k}$.

Equation (41) shows that in the original coordinates the field $\left.\Delta E_{j}\right|_{\xi=0}$ is orthogonal to the ellipsoidal interface (as it must be). On most of the surface, the component along the trail axis, $\Delta E_{1}$, is small, while the two remaining components, $\Delta E_{2,3}$, are usually comparable. The components of electric field parallel and perpendicular to $\boldsymbol{B}$ are given by

$$
\begin{aligned}
& \left.\Delta E_{\|}\right|_{\xi=0}=\left.\Delta E_{3}\right|_{\xi=0} \sin \theta, \\
& \left(\Delta E_{\perp}\right)_{1}=-\left.\Delta E_{3}\right|_{\xi=0} \sin \theta \cos \theta, \\
& \left(\Delta E_{\perp}\right)_{2}=\left.\Delta E_{2}\right|_{\xi=0}, \\
& \left(\Delta E_{\perp}\right)_{3}=\left.\Delta E_{3}\right|_{\xi=0} \cos ^{2} \theta,
\end{aligned}
$$

where, with allowance for Eqs. (8), (12), (34), and (38),

$$
\begin{aligned}
& \left.\Delta E_{3}\right|_{\xi=0}=\frac{\epsilon\left|\Delta E_{X}\right|_{\xi=0}}{\sin \theta} \approx \frac{\epsilon a_{3} x_{1}}{a_{2} x_{3} \Lambda_{1}} \Delta E_{X_{1}}^{(0)}, \\
& \left.\Delta E_{2}\right|_{\xi=0}=\left.\frac{a_{3}^{2} x_{2}}{a_{2}^{2} x_{3}} \Delta E_{3}\right|_{\xi=0} \approx \frac{\epsilon a_{3}^{3} x_{1} x_{2}}{a_{2}^{2} x_{3}^{2} \Lambda_{1}} \Delta E_{X_{1}}^{(0)} .
\end{aligned}
$$

The fact that a significant part of the total polarization electric field on the trail surface is directed along the magnetic field is unusual even for the highly collisional upper D/lower $\mathrm{E}$ regions (large-scale ionospheric electric fields are normally perpendicular to $\boldsymbol{B}$ due to high parallel electron mobility). We will use the boundary electric field in Sects. 5 and 6 when estimating electron heating and the Farley-Buneman instability threshold.

\section{Internal fields and currents; closing of solution}

In this section we consider electric fields and current within the plasma trail. In Sect. 2, we assumed a uniform electric field which leads to uniform internal currents. We will demonstrate that this uniformity is consistent with a constant density trail restricted by an ellipsoidal surface.

If the trail density is much larger than the background plasma density, then the lengthy trail is polarized in such a way that the internal current flows predominantly along the major trail axis $\boldsymbol{X}_{1}, J_{X_{2,3}}^{\text {int }} \ll J_{X_{1}}^{\text {ext }}$. Using Eq. (A15) and neglecting $J_{X_{2,3}}^{\mathrm{int}}$ compared to $J_{X_{1}}^{\mathrm{ext}}$, we obtain

$E_{X_{2}}^{\mathrm{int}} \approx \tilde{q} E_{X_{1}}^{\mathrm{int}}$,

$E_{X_{3}}^{\mathrm{int}} \approx\left(\frac{\psi}{1+\psi}\right)^{1 / 2} E_{X_{2}}^{\mathrm{int}} \cot \theta$,

where $\tilde{q} \equiv q\left[1+\psi \cot ^{2} \theta /(1+\psi)\right]^{-1}$ with $q=\sigma_{\mathrm{H}} / \sigma_{\mathrm{P}}$, see Eq. (A7b). Equation (A15a) then yields

$J_{X_{1}}^{\mathrm{int}} \approx(1+\tilde{q} q) K^{\mathrm{int}} E_{X_{1}}^{\mathrm{int}}$,

where $K^{\text {int }}$ is given by Eq. (A7a) with $N=N^{\text {int }}$. For small $\psi \cot ^{2} \theta$ when $\tilde{q} \approx q$, Ohm's law described by Eq. (46) corresponds to Cowling conductivity, $\sigma_{\mathrm{C}}=\sigma_{\mathrm{P}}+\sigma_{\mathrm{H}}^{2} / \sigma_{\mathrm{P}} \approx \sigma_{\mathrm{H}}^{2} / \sigma_{\mathrm{P}}$.
A more general treatment of 3-D ellipsoidal clouds, $A_{2} \lesssim A_{1}$, shows that the assumption of $J_{X_{2}}^{\text {int }} \ll J_{X_{1}}^{\text {ext }}$, and hence Eqs. (45) and (46), are valid for sufficiently dense trails, $n^{\text {int }} / n^{\text {ext }} \gg A_{2} / A_{3} \sim \Theta_{0}^{-1} \simeq 70$.

Using continuity of the current flow, we relate external ionospheric currents that flow in and out of the trail through the ellipsoidal interface with the internal trail currents. This will allow us to find the still unknown internal electric field, $\boldsymbol{E}^{\mathrm{int}}$, and hence to close the entire solution. Integrating $\nabla \cdot \boldsymbol{J}^{\text {int }}=0$ over a trail cross-section $S_{X_{23}}$ in a $X_{2} X_{3}$-plane at given $X_{1}$, we obtain

$\frac{d I_{X_{1}}^{\text {int }}}{d X_{1}}=-J^{\text {bound }}\left(X_{1}\right)$,

where

$I_{X_{1}}^{\mathrm{int}}\left(X_{1}\right)=\iint J_{X_{1}}^{\mathrm{int}} d S_{X_{2,3}}$

is the total trail current flowing along $X_{1}$, while

$J^{\text {bound }}\left(X_{1}\right)=\oint J_{n}^{\text {bound }} d l_{X_{2,3}}$

is the total external current flowing through the interface per unit length along $X_{1}$. Here $J_{n}^{\text {bound }}$ is the normal component of the boundary current density outside the trail (set positive when directed inward the trail and negative otherwise), $l_{X_{2,3}}$ is the length element of a closed boundary curve corresponding to given $X_{1}$, and the loop integration is taken over this curve. We assumed above that there are no no surface currents, see Fig. 2, so that the normal current density, $J_{n}^{\text {bound }}$, is the same on both sides of the interface.

First, we find the internal current. Since both the plasma density and internal electric field within the trail are assumed uniform, the total trail current flowing along $X_{1}$, i.e., $I_{X_{1}}^{\mathrm{int}}$, is proportional to the trail cross-section, $S_{X_{23}}\left(X_{1}\right)=\pi\left(1-X_{1}^{2} / A_{1}^{2}\right) A_{2} A_{3}$. As a result, we obtain from Eqs. (46) and (48),

$I_{X_{1}}^{\mathrm{int}}=\pi(1+\tilde{q} q)\left(1-\frac{X_{1}^{2}}{A_{1}^{2}}\right) A_{2} A_{3} K^{\mathrm{int}} E_{X_{1}}^{\mathrm{int}}$.

We will use Eqs. (47) and (50) in Sect. 4. Because the internal and external currents on the trail boundary are coupled by Eq. (47) we obtain

$\frac{d I_{X_{1}}^{\mathrm{int}}}{d X_{1}}=-\frac{2 \pi(1+\tilde{q} q) A_{2} A_{3} K^{\mathrm{int}} E_{X_{1}}^{\mathrm{int}} X_{1}}{A_{1}^{2}}$.

On the other hand, for the RHS of Eq. (47), using Eqs. (49), (38), (A6) and the fact that for $A_{3} \ll A_{2}$, Eq. (12), the 2-D elliptical integration path degenerates into two approximately parallel lines nearly perpendicular to $X_{3}$, we obtain

$\oint J_{n}^{\text {bound }} d l_{X_{2,3}} \approx 2 \int_{-A_{2}\left(1-X_{1}^{2} / A_{1}^{2}\right)^{1 / 2}}^{A_{2}\left(1-X_{1}^{2} / A_{1}^{2}\right)^{1 / 2}} J_{X_{3}}^{\text {bound }} d X_{2}$. 
Here the integration limits correspond to $X_{3}=0$, the factor 2 presumes two symmetric contributions of the external current from both positive and negative $X_{3}$, see Fig. 2,

$$
J_{X_{3}}^{\text {bound }} \approx K^{\text {ext }} \frac{A_{3} X_{1} \Delta E_{X_{1}}^{(0)}}{A_{2} X_{3} \Lambda_{1}}
$$

and $K^{\text {ext }}$ is given by Eq. (A7a) with $N=N_{0}$. Recalling the definition of $\Delta E_{X_{1}}^{(0)} \equiv E_{X_{1}}^{(0)}-E_{X_{1}}^{\text {int }}$ and expressing $A_{3} / X_{3}$ from Eq. (10) as $\left(1-X_{1}^{2} / A_{1}^{2}-X_{2}^{2} / A_{2}^{2}\right)^{-1 / 2}$, after integration over $X_{2}$ we obtain

$$
\begin{aligned}
\frac{d I_{X_{1}}^{\mathrm{int}}}{d X_{1}} & =-\oint J_{n}^{\text {bound }} d l_{X_{23}} \\
& \approx-2 \pi K^{\mathrm{ext}} \frac{\left(E_{X_{1}}^{(0)}-E_{X_{1}}^{\mathrm{int}}\right) X_{1}}{\Lambda_{1}}
\end{aligned}
$$

For self-consistency, it is important that both Eqs. (51) and (53) yield the same (linear) dependence on $X_{1}$. This implies that the combination of the water-bag model with the ellipsoidal interface automatically provides the uniformity of the internal electric field (which we used above only as an assumption). From Eqs. (45), (47), (51), and (53), we obtain

$$
E_{X_{1}}^{\mathrm{int}} \approx \frac{E_{X_{1}}^{(0)}}{1+k}, \quad E_{X_{2}}^{\mathrm{int}} \approx \frac{\tilde{q} E_{X_{1}}^{(0)}}{1+k}, \quad\left|E_{X_{3}}^{\mathrm{int}}\right| \ll\left|E_{X_{1,2}}^{\mathrm{int}}\right| .
$$

where

$$
\begin{aligned}
k & \approx \frac{(1+\tilde{q} q) \Lambda_{1} A_{2} A_{3}}{A_{1}^{2}}\left(\frac{N^{\mathrm{int}}}{N_{0}}\right) \\
& \approx \frac{(1+\tilde{q} q) \Lambda_{1}(1+\psi)^{1 / 2} \Theta_{0} a_{2} a_{3}}{a_{1}^{2} \sin ^{3} \theta}\left(\frac{n^{\mathrm{int}}}{n_{0}}\right) .
\end{aligned}
$$

In obtaining Eq. (55), we used Eq. (11). The internal electric field is determined by the external field component $E_{X_{1}}^{(0)}$ because only this component creates a large potential difference along the extended trail. The internal field component along the trail, $E_{X_{1}}^{\mathrm{int}}$, is always less than $E_{X_{1}}^{(0)}$, while the transverse component, $E_{X_{2}}^{\text {int }}$, which is $\tilde{q}$ times larger than $E_{X_{1}}^{\text {int }}$, can be either larger or smaller than $E_{X_{1}}^{(0)}$.

The physical mechanism behind the formation of the internal field is as follows. The external electric field $E_{X_{1}}^{(0)}$ initially penetrates to the highly conducting trail and immediately gives rise to a strong Hall current along $x_{2}$. This current quickly accumulates opposite charges on the opposite edges of the dense trail. As a result, a strong internal field starts building up in the $x_{2}$-direction until it creates a sufficient Pedersen current to counterbalance the Hall current. This, much stronger, field gives rise to a secondary Hall current in the direction perpendicular to both $x_{2}$ and $\boldsymbol{B}$ - a process equivalent to the electrojet formation. The $x_{3}$-component of the secondary Hall current (if $\theta \neq 90^{\circ}$ ) polarizes the trail in this direction, resulting in a final current directed mainly along the trail. The trail is long but restricted in the $x_{1}$-direction, so that the secondary Hall current polarizes the trail in this direction as well. However, this polarization is smoothly distributed along the trail length. It partially expels the major component of the external field, $E_{1}^{(0)}$, from the trail until the eventual reduced field in the $x_{1}$-direction provides a self-consistent quasi-stationary closure of the trail current through the much rarer ionosphere. The denser the trail is, the stronger field expulsion is. Estimates show that the fields and currents will develop with a few $\mu$ s, i.e., much faster than the meteor trail evolution. This means that as soon as the extended trail starts forming, it is already polarized with the quasi-stationary distribution of fields and currents.

Equation (54) gives the explicit expression for the internal electric field, $\boldsymbol{E}_{\boldsymbol{X}}^{\text {int }}$, in terms of the external field, $\boldsymbol{E}_{X_{1}}^{(0)}$. This closes the solution for spatial distribution of the electrostatic potential and currents in the entire space, see Sect. 3. In particular, using

$\Delta E_{X_{1}}^{(0)}=E_{X_{1}}^{(0)}-E_{X_{1}}^{\mathrm{int}} \approx \frac{k E_{X_{1}}^{(0)}}{1+k}$

and Eq. (52), we obtain on the trail-ionosphere interface the major component of the external current density that flow in or out of the dense trail,

$$
J_{X_{3}}^{\text {bound }} \approx K^{\text {ext }} \frac{k A_{3} X_{1} E_{X_{1}}^{(0)}}{(1+k) A_{2} X_{3} \Lambda_{1}} .
$$

For very dense trails where $k \gg \tilde{q} \gg 1$, the process of field expulsion is so efficient that the total internal electric field becomes much smaller than the external field. Observations show that this type of dense trails occur frequently. Consider, e.g., a purely vertical trail $\left(\theta=90^{\circ}\right)$ in the nighttime equatorial ionosphere, where $\theta=90^{\circ}, \tilde{q}=q \gg 1$, and $n_{0} \sim 10^{8} \mathrm{~m}^{-3}$. According to Eqs. (55) and (A7b), dense trails require that $n^{\text {int }} / n_{0} \gg[(1+\psi) / \psi]^{1 / 2} a_{1}^{2} /\left(\Lambda_{1} a_{2} a_{3}\right)$. This imposes a lower restriction on the electron line density of a dense trail, $N_{\text {lin }}=\pi n^{\text {int }} a_{2} a_{3}$. For altitudes below $95 \mathrm{~km}(\psi>1)$ and $\pi / \Lambda_{1} \sim 1$, a dense trail must have $N_{\text {lin }} \gg a_{1}^{2} n_{0}$. For $a_{1} \sim 1 \mathrm{~km}$, this requires $N_{\operatorname{lin}} \gg 10^{14} \mathrm{~m}^{-1}$. While the majority of radarobserved trails have $N_{\text {lin }} \sim 10^{14} \mathrm{~m}^{-1}$, recent Jicamarca radar observations show that trails with $N_{\text {lin }} \sim 10^{15} \mathrm{~m}^{-1}$ are common (E. Bass, private communication). At daytime, when the typical background ionosphere density is about two orders of magnitude larger, we obtain a much stronger restriction, $N_{\text {lin }} \gg 10^{16} \mathrm{~m}^{-1}$. For higher altitudes $(\psi \ll 1)$, longer trails, or smaller $\theta$, very dense trails require higher $N_{\text {lin }}$ values. Trails with $N_{\text {lin }} \sim 10^{16} \mathrm{~m}^{-1}$ occur on some rare occasions and even $N_{\text {lin }} \gtrsim 10^{17} \mathrm{~m}^{-1}$ may appear.

For more typical, less dense trails that satisfy $\tilde{q} \gtrsim k \gg 1$, i.e., for $a_{1}^{2} n_{0} / q \ll N_{\text {lin }} \lesssim a_{1}^{2} n_{0}$, the component of the internal field 
along the major trail axis, $E_{X_{1}}^{\mathrm{int}}$, is much smaller than the corresponding component of the external field, $E_{X_{1}}^{(0)}$, while the transverse component, $E_{X_{2}}^{\text {int }}$, and hence the total internal field, are larger than $E_{X_{1}}^{(0)}$. For even lower-density trails where $k \ll 1$, i.e., for $N_{\text {lin }} \ll a_{1}^{2} n_{0} / q$, the process of field expulsion becomes inefficient, $E_{X_{1}}^{\text {int }} \approx E_{X_{1}}^{(0)}$, while the transverse

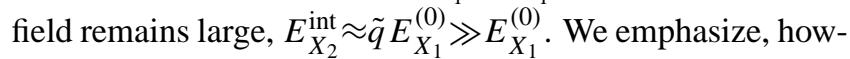
ever, that even the last case applies only to sufficiently dense trails, $n^{\text {int }} / n_{0} \gg 70$, as we discussed in the passage following Eq. (46). For trails of a much lower density, $n^{\text {int }} / n_{0} \lesssim 70$, the transverse electric field decreases with trail density until it gradually disappears as $n^{\text {int }}$ approaches $n_{0}$. In this paper, however, we are not interested in such low-density regimes.

The current densities and internal electric fields have been expressed in terms of $E_{X_{1}}^{(0)}$. To express them in terms of the original $\boldsymbol{E}^{(0)}$, we can apply Eqs. (A11) and (A12). Simple reasoning shows that $E_{X_{1}}^{(0)} \approx E_{13}^{(0)}$, where $E_{13}^{(0)}$ is the full component of the external field, $\boldsymbol{E}^{(0)}$, in the $x_{1} x_{3}$-plane. Since the external field is perpendicular to $\boldsymbol{B}$, the first rotation to the coordinate system aligned with $\boldsymbol{B}$, results in $E_{X_{1}^{\prime}}^{(0)}=E_{13}^{(0)}$. The compression of the coordinates along $X_{3}^{\prime}$ by the small factor $\epsilon$ does not affect the field because $E_{X_{3}^{\prime}}^{(0)}=-\partial \Phi / \partial X_{3}^{\prime}=0$. The final rotation through the small angle $\chi$ yields

$E_{X_{1}}^{(0)}=E_{13}^{(0)} \cos \chi \approx E_{13}^{(0)}$

with $\left|E_{X_{3}}^{(0)}\right|=\left|E_{13}^{(0)}\right| \sin \chi \ll\left|E_{X_{1}}^{(0)}\right|$. One can derive Eq. (58) directly from Eqs. (A11a) and (A12a) by expressing the external electric field, $\boldsymbol{E}^{(0)} \perp \boldsymbol{B}$, as $E_{1}^{(0)}=E_{13}^{(0)} \sin \theta$ and $E_{3}^{(0)}=-E_{13}^{(0)} \cos \theta$. In particular, according to Eqs. (43), (44a), (56), and (58), on the trail boundary the electric field component parallel to $\boldsymbol{B}$ is given by

$\left.\Delta E_{\|}\right|_{\xi=0} \approx \frac{\epsilon k a_{3} x_{1} E_{13}^{(0)} \sin \theta}{(1+k) a_{2} x_{3} \Lambda_{1}}$,

where $k$ is defined by Eq. (55).

Now we estimate the total current that flows through the trail. Combining Eqs. (50), (54) and (58) with Eqs. (8), (11), (A7), and the definition of $\tilde{q}$, we obtain

$$
\begin{aligned}
I_{X_{1}}^{\mathrm{int}} & \approx\left(1+\frac{\psi \cot ^{2} \theta}{1+\psi}\right)\left(1-\frac{x_{1}^{2}}{a_{1}^{2}}\right) \\
& \times \frac{\pi \sqrt{\psi} a_{2} a_{3} e n^{\mathrm{int}} E_{13}^{(0)}}{(1+\psi) \Theta_{0}(1+k) B \sin \theta} .
\end{aligned}
$$

The trail current is proportional to the trail cross-section, so that its maximum, $I_{\max }^{\mathrm{int}}$, is reached at the trail center, $x_{1}=0$. For small $k$, Eq. (55), the trail current is determined by the external field which nearly fully penetrates into the trail. This current grows in proportion to the trail plasma density. In the opposite limit of $k \gg 1$, as we can obtain directly from Eqs. (50) and (55), the maximum trail current is given by

$$
\begin{aligned}
I_{\max }^{\mathrm{int}} & \approx \frac{\pi a_{1}^{2} \sin ^{2} \theta}{\Lambda_{1}}\left(\frac{1+\psi}{\psi}\right)^{1 / 2} \frac{e n_{0} E_{13}^{(0)}}{B} \\
& \approx 0.1 \mathrm{~A}\left(\frac{1+\psi}{\psi}\right)^{1 / 2} \frac{\sin ^{2} \theta}{\Lambda_{1}}\left(\frac{n_{0}}{10^{10} \mathrm{~m}^{-3}}\right) \\
& \times \frac{5 \times 10^{4} \mathrm{nT}}{B}\left(\frac{E_{13}^{(0)}}{1 \mathrm{mV} / \mathrm{m}}\right)\left(\frac{a_{1}}{1 \mathrm{~km}}\right)^{2},
\end{aligned}
$$

where we have normalized all dimensional parameters to typical numerical values to make practical calculations simple. In this limit, the maximum current is independent of the trail plasma density but is proportional to the background ionosphere density, $n_{0}$. This is not surprising because for $k \gg 1$ the external electric field $E_{13}^{(0)}$ penetrates into the trail only weakly. This maximum trail current is determined by the ionospheric currents collected from a large volume around the nearly equipotential trail. It depends on the small scale $a_{2}$ logarithmically weakly, via $\Lambda_{1} \approx \ln \left(4 a_{1} \sin \theta / a_{2}\right)-1$, see Eqs. (11) and (32). There is no dependence on $a_{3}$ because the ionospheric currents that flow into and out of the trail are dominated by electron currents parallel to $\boldsymbol{B}$, so that only the trail cross-section perpendicular to $\boldsymbol{B}$ plays a role. Equation (61) shows that for quite realistic trail and ionosphere parameters, $a_{1} \gtrsim 3 \mathrm{~km}, n_{0} \gtrsim 10^{10} \mathrm{~m}^{-3}, E_{12}^{(0)} \gtrsim 10 \mathrm{mV} / \mathrm{m}, \psi \ll 1$, the total short-circuiting current through the trail can reach many amperes.

\section{$5 \quad$ Heating of near-trail plasma}

The strong electric field induced in the near-trail region, especially its component parallel to $\boldsymbol{B}$, can energize electrons. Due to pitch-angle scattering caused by frequent collisions with neutrals, such energization is efficiently spread over all angles in the velocity space, resulting in nearly isotropic electron heating. Indeed, for electrons with energies below $2 \mathrm{eV}$, the average fraction of electron energy loss during one inelastic or elastic collision with neutrals, $\delta_{e n}$, is small since in this energy range colliding electrons cannot excite efficiently vibrational and electronic levels of neutral molecules. At the same time, after the collisional impact they easily change the direction of motion, so that the electron velocity distribution becomes nearly isotropic with the small mean directional speed, $V_{e} \equiv\left|\boldsymbol{V}_{e}\right| \sim \delta_{e n}^{1 / 2} v_{T e} \ll v_{T e}$, where $v_{T e}=\left(T_{e} / m_{e}\right)^{1 / 2}$ (Gurevich, 1978). If the parallel electric field is so strong that $V_{e}$ substantially exceeds the initial mean thermal speed, $v_{T e 0}=\left(T_{e 0} / m_{e}\right)^{1 / 2}$, then, during a short time $\sim\left(\delta_{e n} v_{e n}\right)^{-1}$, the electron population heats up in such a way that $V_{e} \ll v_{T e}\left(T_{e}\right)$ becomes valid. While the angular velocity distribution of electrons becomes nearly isotropic, their energy distribution can differ significantly 
from Maxwellian (Gurevich, 1978; Milikh and Dimant, 2003).

We will estimate electron heating assuming local energy balance with no heat transport,

$\frac{3}{2} \frac{d T_{e}}{d t}=m_{e} v_{e n} V_{e}^{2}-\frac{3}{2} \delta_{e n} v_{e n}\left(T_{e}-T_{e 0}\right)$.

The first term of the RHS describes frictional heating, while the second term describes collisional cooling against neutrals with the temperature $T_{n} \approx T_{e 0}$. Under stationary conditions, taking into account electron heating by the electric field parallel to $\boldsymbol{B}$ only and $m_{e} v_{e n} V_{e}^{2} \approx j_{e \|}^{2} / n_{0} \sigma_{e \|}=m_{e} v_{e n} j_{e \|}^{2} / n_{0}^{2} e^{2}$, we obtain

$\Delta T_{e} \equiv T_{e}-T_{e 0}=\frac{2 m_{e} j_{e \|}^{2}}{3 \delta_{e n} n_{0}^{2} e^{2}}$,

where $j_{e \|}=e n_{0} \Delta E_{\|} /\left(m_{e} v_{e n}\right)$ is the parallel current density. Combining Eqs. (6) and (59) with $v_{e n}=\Omega_{e} \Theta_{0} \sqrt{\psi}$, we obtain

$\left.j_{e \|}\right|_{\xi=0} \approx \frac{k(1+\psi)^{1 / 2} a_{3} x_{1}}{(1+k) \psi^{1 / 2} a_{2} x_{3} \Lambda_{1}} \frac{n_{0} e E_{13}^{(0)} \sin \theta}{B}$.

As a result, on the trail boundary, we have

$$
\begin{aligned}
& \left.\Delta T_{e}\right|_{\xi=0}=\frac{2 m_{e}(1+\psi)}{3 \delta_{e n} \psi}\left[\frac{k a_{3} x_{1} E_{13}^{(0)} \sin \theta}{(1+k) a_{2} x_{3} \Lambda_{1} B}\right]^{2} \\
& \approx 7 \times 10^{-3} \mathrm{~K}\left(\frac{E_{13}^{(0)} \sin \theta}{1 \mathrm{mV} / \mathrm{m}}\right)^{2} \frac{2.5 \times 10^{-3}}{\delta_{e n}} \\
& \times \frac{1+\psi}{\psi}\left(\frac{5 \times 10^{4} \mathrm{nT}}{B}\right)^{2}\left[\frac{k a_{3} x_{1}}{(1+k) a_{2} x_{3} \Lambda_{1}}\right]^{2} .
\end{aligned}
$$

Equation (65) shows that electron heating near the trail can be quite significant, especially at larger altitudes where $\psi \ll 1$. Assuming, e.g., $\psi=0.01$ (this corresponds to an altitude $\simeq 110 \mathrm{~km}$ at the geomagnetic equator and $\simeq 107 \mathrm{~km}$ at high latitudes), $x_{1} \simeq a_{1}, \quad x_{3} \lesssim a_{3}$, $a_{1} / a_{3}=10^{3}, \quad k|\sin \theta \cos \chi| /(1+k) \Lambda_{1}=0.1$, and choosing typical conditions for the equatorial $\mathrm{E}$ region, $E_{13}^{(0)}=5 \mathrm{mV} / \mathrm{m}$, $B \approx 2.5 \times 10^{4} \mathrm{nT}$, we obtain $\Delta T_{e} \gtrsim 7 \times 10^{4} \mathrm{~K}$. At high latitudes with much stronger electric fields, one would expect even stronger heating effects. However, due to excitation of vibrational levels for $T_{e} \gtrsim 1 \mathrm{eV}$, nonlinear and nonlocal effects associated with the temperature dependence of $v_{e n}$ and heat conductivity, the maximum temperature should saturate at a lower level. Note also that the elevated electron temperatures, which can penetrate inside the trail, can result in faster trail diffusion (but only for a restricted time because the heating reduces in proportion to $\left.a_{2}^{-2} \propto t^{-1}\right)$. One more possible effect of the short-time temperature elevation in the $\mathrm{eV}$ temperature range could be an increased airglow in the nighttime ionosphere. We discuss this in the next section. The strong electric field induced around the trail can also heat ions, albeit not as dramatically as electrons because the ion isotropic mobility in the upper D/lower E regions is $\Theta_{0}^{-2} \approx 5500$ times less than the electron parallel mobility.

We should bear in mind, however, that the average fraction of collisional energy loss, $\delta_{e n}$, and the electron-ion collision frequency, $v_{e n}$ (involved in parameters $\psi$ and $k$ ), are temperature-dependent. The temperature dependence of $\delta_{e n}$ is rather complicated and is sensitive to the shape of the electron velocity distribution (Gurevich, 1978; Milikh and Dimant, 2003). According to kinetic calculations, $\delta_{e n}$ is roughly constant between $T_{e} \simeq 2000 \mathrm{~K}$ and $T_{e} \simeq 10^{4} \mathrm{~K}$ and then starts increasing sharply at larger temperatures (Gurevich, 1978, Fig. 10). Due to this, we do not expect electron temperatures be well above $1 \mathrm{eV}$. At smaller temperatures, we can approximate $\delta_{e n}\left(T_{e}\right)$ by a constant value, $\delta_{e n} \approx 2.5 \times 10^{-3}$. Further, due to the temperature dependence of the electron-neutral collision frequency, strong electron heating can modify the ionospheric background plasma, making the entire problem nonlinear and much more complicated. For reasonable estimates, however, we can use the above expressions with the undisturbed value of $v_{e n}$. The reason is that ionospheric currents responsible for heating are determined by the charge conservation and the flow geometry of the current collection from a large volume of mainly undisturbed ionosphere located well beyond the trail. To some extent, this can mitigate the sensitivity of the currents to local temperature variations. A more accurate self-consistent treatment requires 3-D simulations which we plan to perform in the future.

\section{Discussion}

Our analysis shows that sufficiently dense and long meteor plasma trails in an external DC electric field result in a number of potentially observable features. The amplified electric field can drive plasma instabilities in the near-trail ionosphere, which complements similar effects within the trail caused by the ambipolar fields associated with the trail diffusion. The electric field near the trail can acquire a significant component parallel to the magnetic field. Because this is the direction of high electron mobility, the parallel electric field can locally heat ionospheric electrons to the temperatures in the $1 \mathrm{eV}$ range. At nighttime, at sufficiently high altitudes $\sim 110 \mathrm{~km}$, such heating may result in increased atmospheric airglow. The dramatic redistribution of the electric potential near the trail will drive currents that may exceed $1 \mathrm{~A}$ across the trail, see Eq. (61). The energy for these strong effects is provided by the ionospheric dynamo that sustains the external DC field, rather than by the original meteoroid energy. We expect these effects to occur in the equatorial and highlatitude E regions, where the external field is strong. Similar, but less intense, processes may also take place at midlatitudes where the typical values of the DC electric field are 
$\sim 1-2 \mathrm{mV} / \mathrm{m}$. Also, strong neutral winds perpendicular to $\boldsymbol{B}$ can cause similar effects. Below we make some estimates of possible physical processes associated with strong induced electric fields, currents, and electron heating.

\subsection{Farley-Buneman instability threshold}

Radars observe meteor instabilities in the form of nonspecular echoes. These instabilities are driven by strong electric fields that develop within or nearby meteor plasma trails. The fields associated with trail ambipolar diffusion are mainly located within the trails (Dimant and Oppenheim, 2006a,b). The strong fields induced by external fields can significantly modify the threshold conditions for instabilities in the near-threshold regions.

Now we discuss the threshold conditions for the FarleyBuneman (FB) instability, which is the most robust E-region plasma instability. The FB instability is driven by a sufficiently strong electric field perpendicular to the magnetic field, $\boldsymbol{E}_{\perp}$, via the electron Hall velocity, $\boldsymbol{V}_{\mathrm{Hall}}=\boldsymbol{E}_{\perp} \times \boldsymbol{B} / B^{2}$, and can only be excited provided $V_{\text {Hall }} \equiv\left|\boldsymbol{V}_{\text {Hall }}\right|>(1+\psi) C_{s}$, where $C_{s}=\left[\left(\gamma_{e} T_{e}+\gamma_{i} T_{i}\right) / m_{i}\right]^{1 / 2}$ is the ion-acoustic speed. Everywhere near the trail, except near $x_{3}=0$, the components parallel and perpendicular to $\boldsymbol{B}$ of the near-trail electric field are comparable, $\Delta E_{\|} \sim \Delta E_{\perp}$.

If a strong perpendicular field is sufficient to drive the instability in the undisturbed ionosphere, a comparable parallel field also exists to simultaneously heat the plasma and, hence, to increase the FB instability threshold. On the other hand, the temperature dependence of the electron-neutral collision frequency causes the induced electric field to increase in the heated region near the trail. The resultant threshold conditions can be aggravated or improved, depending on the balance between these factors. Below we discuss only the induced electric field beyond the trail as the driving force for the instability, while the internal trail field can also be of importance.

Assuming sufficiently strong electron heating, $T_{e} \gg T_{e 0}, T_{i}$, and isothermal conditions, $\gamma_{e, i}=1$, we obtain that the FB threshold electric field amplitude is $E_{\mathrm{Thr}}\left(T_{e}\right) \approx\left(1+\psi\left(T_{e}\right)\right)\left(T_{e} / 2 T_{e 0}\right)^{1 / 2} E_{\mathrm{Thr}}^{\min }\left(T_{e 0}\right)$, where $E_{\mathrm{Thr}}\left(T_{e 0}\right)$ is the minimum threshold field for the undisturbed temperature $T_{e 0} \approx T_{i 0}$,

$E_{\mathrm{Thr}}^{\min }=\sqrt{\frac{2 T_{e 0}}{m_{i}}} B \approx 20 \frac{\mathrm{mV}}{\mathrm{m}}\left(\frac{B}{5 \times 10^{4} \mathrm{nT}}\right)\left(\frac{T_{e 0}}{300 \mathrm{~K}}\right)^{1 / 2}$.

The parallel current near the trail surface, $j_{e \|}$, is collected from a large ionospheric volume and is determined mainly by the macroscopic electrodynamic structure. We expect it to be less susceptible to local heating than to the local electric field. Neglecting completely the effect of heating on $j_{e \|}$, we can find the modified parallel component of local induced electric field via $\Delta E_{\|}=m_{e} v_{e n}\left(T_{e}\right) j_{\|} /\left(n_{0} e^{2}\right)$, where $\left.j_{\|}\right|_{\xi=0}$ is given by Eq. (64). The perpendicular component, $\Delta E_{\perp}$, should change in proportion to $\Delta E_{\|}$because near the trail the total induced field always remains perpendicular to the trail surface,

$\Delta E_{\perp}=\frac{x_{2} \Delta E_{\|}}{x_{3}}=\frac{x_{2} m_{e} v_{e n}\left(T_{e}\right) j_{\|}}{x_{3} n_{0} e^{2}}$.

Assuming for $v_{e n}\left(T_{e}\right)$ the power-law approximation, $v_{e n} \approx v_{e n 0}\left(T_{e} / T_{e 0}\right)^{5 / 6}$ (Gurevich, 1978) and neglecting ion heating, we obtain

$$
\frac{\Delta E_{\perp}\left(T_{e}\right)}{E_{\mathrm{Thr}}\left(T_{e}\right)} \simeq \frac{\sqrt{2}\left(T_{e} / T_{e 0}\right)^{-1 / 12} \Delta E_{\perp}\left(T_{e 0}\right)}{\left[\left(1+\psi\left(T_{e}\right)\right)\left(1+\psi\left(T_{e 0}\right)\right)\right]^{1 / 2} E_{\mathrm{Thr}}^{\min }\left(T_{e 0}\right)} .
$$

This relation shows that strong electron heating slightly affects the conditions of FB instability generation, mainly through the parameter $\psi\left(T_{e}\right) \propto T_{e}^{5 / 6}$, when it becomes $\gtrsim 1$. Expressing all quantities in Eq. (67) in terms of $E_{13}^{(0)}$, with allowance for Eqs. (64), (65), and (66), we verify that for $x_{j} \sim a_{j}$ and sufficiently large ratio $a_{1} / a_{2}$ the FB instability near the trail surface can be excited by the induced electric field even if the external electric field is well below the FB threshold (for example, for $E_{13}^{(0)} \sim 1-2 \mathrm{mV} / \mathrm{m}$, which is typical for midlatitudes). Note also that the same pertains to the region inside the trail under conditions of moderately dense trail $1 \ll k \lesssim \tilde{q}, n^{\text {int }} / n_{0} \gg 70$, when the transverse component of the internal field, $E_{X_{2}}^{(0)}=E_{x_{2}}^{(0)}$, is approximately $\tilde{q}$ times the external field $E_{X_{1}}^{(0)} \approx E_{13}^{(0)}$ (see the comment below Eq. 57).

\subsection{Airglow caused by the electron heating in the me- teor trail}

In this section, we discuss when meteor-heated electrons generate a detectable airglow in the nighttime ionosphere. We consider excitation of three major electronic levels $\mathrm{O}\left({ }^{1} \mathrm{~S}\right)$, $\mathrm{N}_{2}\left(\mathrm{~B}^{3} \Pi_{g}\right)$ and $\mathrm{N}_{2}\left(\mathrm{C}^{3} \Pi_{u}\right)$ by electron impacts. The excitation leads to the emissions of the oxygen green line $\lambda=557.7 \mathrm{~nm}$ and of $\mathrm{N}_{2}(1 \mathrm{P})$ and $\mathrm{N}_{2}(2 \mathrm{P})$ bands. The nitrogen first positive system emits in the band of $(478-2531) \mathrm{nm}$, while the second positive system emits in the band of (268546) $\mathrm{nm}$ (McEwan and Phillips, 1975). The oxygen redline emission, although having a low excitation threshold, is strongly quenched out below $130 \mathrm{~km}$ and thus can be neglected.

Let us estimate the luminous flux for a given transition $m$, $I^{m}$, in Rayleighs (R), using the following equation

$I^{m}(\mathrm{R})=\frac{10^{-6}}{4 \pi\left(1+\tau_{\mathrm{life}} k_{q} N_{n}\right)} \int k_{e x}^{m} n_{e} N_{n}^{m} d x$,

where the denominator shows effect of the collisional quenching. Here $\tau_{\text {life }}$ is the lifetime of the excited electronic level, $k_{q}$ is its quenching rate due to collisions with the neutral species having the number density $N_{n}$. Furthermore, $k_{e x}^{m}$ 

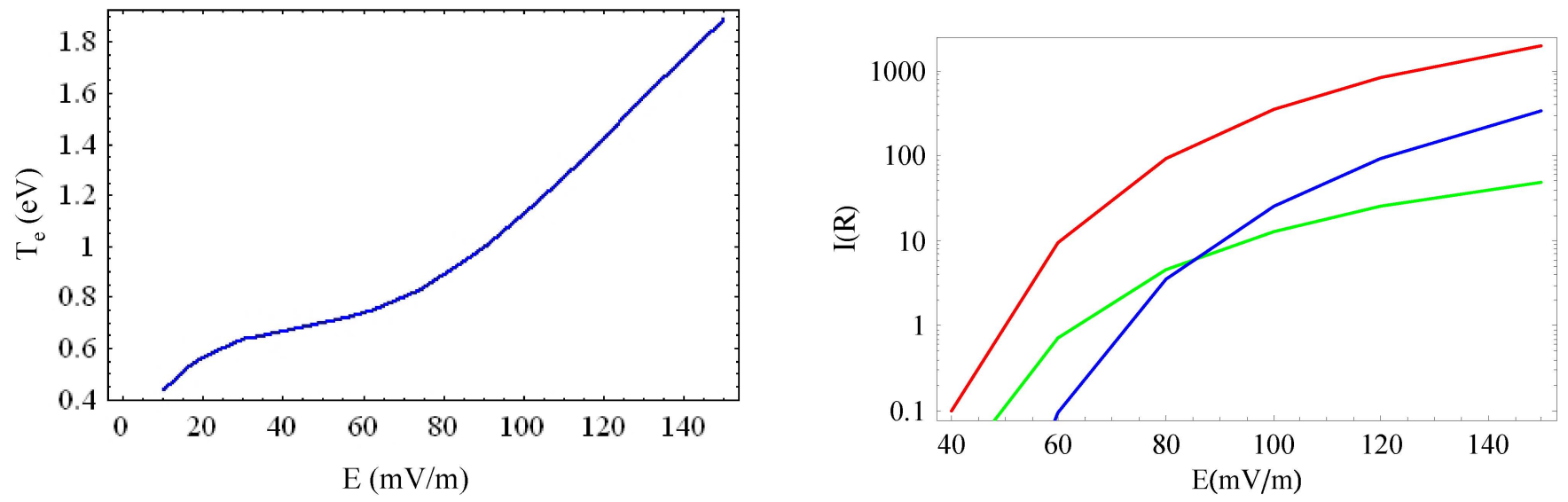

Fig. 4. Electron temperature (left panel) and luminous flux versus parallel electric field (right panel). The green trace corresponds to the oxygen green-line emission; the red trace corresponds to $\mathrm{N}_{2}(1 \mathrm{P})$; and the blue trace corresponds to $\mathrm{N}_{2}(2 \mathrm{P})$ band emission.

is the excitation rate of the electron level considered by the electrons of density $n_{e}, N_{n}^{m}$ is the density of the respective neutral species. The integration is conducted along the line of sight of the ground-based optical detector.

The excitation rate of the above electronic levels of $\mathrm{O}$ and $\mathrm{N}_{2}$ was calculated by using a kinetic code. The calculation of electron heating by the electric field follows the well-established procedure of ionospheric and atmospheric breakdown (Gurevich et al., 1997; Tsang et al., 1991; Papadopoulos et al., 1993). The computer code employed in the present work is based on solving a local kinetic equation that describes the evolution of the time-averaged electron distribution function (EDF), $f_{e}(\varepsilon, t), \varepsilon \equiv m_{e} v_{e}^{2} / 2$, in the presence of a constant ambient electron field parallel to $\boldsymbol{B}_{0}$ with the amplitude $E=\Delta E_{\|}$, and various kinds of inelastic electronneutral collisions. According to Eq. (59), for values of the external electric field reaching tens to hundred of $\mathrm{mV} / \mathrm{m}$, depending on the ratio $a_{1} / a_{3}$ and the specific position on the trail boundary, the parallel field can also reach in some cases tens of $\mathrm{mV} / \mathrm{m}$ or even more.

We conducted our computations for the neutral density and compositions which corresponds to the height of $110 \mathrm{~km}$, the model of IRI was applied $\left(65^{\circ} \mathrm{N}\right.$, winter $)$. The excitation rates were obtained by our code as $k_{e x}^{m}=\int_{\varepsilon_{e x}^{m}}^{\infty} \sigma_{e x}^{m} f_{e}(\varepsilon) \sqrt{\varepsilon} d \varepsilon$, where $\varepsilon_{e x}^{m}$ is the excitation threshold and $\sigma_{e x}^{m}$ is the excitation cross section.

Figure 4 shows the luminous flux for the green-line of oxygen, as well as the $\mathrm{N}_{2}(1 \mathrm{P})$ and $\mathrm{N}_{2}(2 \mathrm{P})$ emissions as functions of the parallel electric field $E$. It was computed for the typical trail conditions, $n_{e}=10^{4} \mathrm{~cm}^{-3}$, and the $100 \mathrm{~m}$ total length along the line of sight. Note that collisional quenching of $\mathrm{O}\left({ }^{1} \mathrm{~S}\right)$ by atomic oxygen with $\tau_{\text {life }}=0.8$ s for $\mathrm{O}\left({ }^{1} \mathrm{~S}\right)$ and $k_{q}=7.5 \times 10^{-12} \mathrm{~cm}^{3} / \mathrm{s}$ (McEwan and Phillips, 1975) was taken into account.
For two other electronic levels $\mathrm{N}_{2}(\mathrm{~B})$ and $\mathrm{N}_{2}(\mathrm{C})$, having the lifetimes $\sim 1 \mu \mathrm{s}$ (B) and a fraction of $\mu \mathrm{s}(\mathrm{C})$, the collisional quenching at $110 \mathrm{~km}$ is negligible. The figure reveals that for parallel electric field in excess of $80 \mathrm{mV} / \mathrm{m}$ the expected emissions can be detected by a ground-based telescope. And since the emission of interest lasts for about a second after the tip of a meteor passes, it is possible to detect such emission by using a sensitive camera triggered by a strong signal due to the burning meteoroids, with the total observation duration up to $1 \mathrm{~s}$.

During the nighttime, the background emission of the green line $557.7 \mathrm{~nm}$ is of about $300 \mathrm{R}$ (McEwan and Phillips, 1975 ) in the absence of precipitating charged particles. This emission originates from two altitudes: $\sim 250 \mathrm{~km}$ and $\sim 90 \mathrm{~km}$. At the higher altitude, it is generated by electron ion recombination, while at the lower height it is produced by triple collisions of atomic oxygen.

As far as $\mathrm{N}_{2}(1 \mathrm{P})$ and $\mathrm{N}_{2}(2 \mathrm{P})$ emissions are concerned, in the absence of particle precipitations, they are not generated during the nighttime. However, since they are broad-band emissions they overlap with some other background emissions. Therefore the best way to detect the artificial airglow of meteor trails is to focus the optical detector on some parts of the $\mathrm{N}_{2}(1 \mathrm{P})$ and $\mathrm{N}_{2}(2 \mathrm{P})$ spectrum in which such overlap with background emission is weak. In the latter case, a few $\mathrm{R}$ emission in a bandwidth of 10-20 nm should be detectable. We note, however, that strong electric fields at high latitudes are often accompanied by bright auroras. This may forbid observations of weak emissions associated with electron heating near meteor trails.

We also computed the rate of dissociative attachment to $\mathrm{O}_{2}$ along with the impact ionization rate. It was found that for $E \leq 150 \mathrm{mV} / \mathrm{m}$, the ionization rate was smaller than the attachment rate. Thus, the field $E \leq 150 \mathrm{mV} / \mathrm{m}$ is under the ionization threshold, while at $110 \mathrm{~km}$ the attachment rate for 
$E=100-150 \mathrm{mV} / \mathrm{m}$ ranges between 2 and $5.5 \mathrm{~s}^{-1}$. For emissions that last longer than $0.1 \mathrm{~s}$ the electron density reduction due to attachment to $\mathrm{O}_{2}$ becomes noticeable.

\section{Conclusions}

We have developed an analytical 3-D theoretical model of the interaction of an external DC electric field with a dense meteor plasma trail. This model predicts that such interaction will result in a distortion of the external field causing a large amplification of the field near the trail edges. The meteor will also drive currents through itself and into the surrounding ionosphere, short-circuiting the local ionosphere.

Unlike the ambient ionospheric DC electric field which points perpendicular to $\boldsymbol{B}_{0}$, the induced electric field near the trail surface will have comparable components perpendicular and parallel to $\boldsymbol{B}_{0}$. The perpendicular electric field component can be strong enough to generate plasma instabilities near the trail edges. These instabilities often result in an increased level of electron density irregularities visible to high-power large-aperture radars as non-specular echoes. The strong component of the electric field parallel to the magnetic field can result in a significant (up to $\sim 1 \mathrm{eV}$ ) heating of electrons. In some cases, such heating should be measurable via the increased airglow.

\section{Appendix A}

\section{Coordinate transformations}

In this appendix we describe a chain of coordinate transformations that result in Laplace's Eq. (9) and the renormalized ellipsoidal interface given by Eq. (10).

First, we perform a coordinate rotation to a new cartesian system $x_{1,2,3}^{\prime}$ where $x_{3}^{\prime}$ is along $\boldsymbol{B}, x_{2}^{\prime}=x_{2}$, while $x_{1}^{\prime}$ becomes perpendicular to both $x_{2}^{\prime}$ and $x_{3}^{\prime}$,

$x_{1}=x_{1}^{\prime} \sin \theta+x_{3}^{\prime} \cos \theta$,

$x_{2}=x_{2}^{\prime}$,

$x_{3}=-x_{1}^{\prime} \cos \theta+x_{3}^{\prime} \sin \theta$.

Rotation is a unitary transformation, $\partial x_{i} / \partial x_{k}^{\prime}=\partial x_{k}^{\prime} / \partial x_{i}$, so that both covariant and contravariant vectors transform as $x_{i}$. The covariant electric field in the rotated ("primed") system, $\boldsymbol{E}^{\prime}=-\nabla^{\prime} \Phi$, relates to the electric field in the original coordinates, $\boldsymbol{E}$, as

$E_{1}^{\prime}=E_{1} \sin \theta-E_{3} \cos \theta$,

$E_{2}^{\prime}=E_{2}$,

$E_{3}^{\prime}=E_{1} \cos \theta+E_{3} \sin \theta$,

For a plasma with the given uniform density $n$, Eq. (2) with $\hat{x}_{3}^{\prime} \| \boldsymbol{B}$ yields the following "primed" components of the total electric current density:

$j_{1}^{\prime}=\sigma_{\mathrm{P}} E_{1}^{\prime}+\sigma_{\mathrm{H}} E_{2}^{\prime}$,

$j_{2}^{\prime}=-\sigma_{\mathrm{H}} E_{1}^{\prime}+\sigma_{\mathrm{P}} E_{2}^{\prime}$,

$j_{3}^{\prime}=\sigma_{\|} E_{3}^{\prime}$,

where

$$
\begin{aligned}
\sigma_{\mathrm{P}} & \approx \frac{n e^{2}}{m_{i} v_{i n}}+\frac{n e^{2} v_{e n}}{m_{e} \Omega_{e}^{2}}=\frac{(1+\psi) \Theta_{0} n e}{B \sqrt{\psi}}, \\
\sigma_{\mathrm{H}} & \approx \frac{n e}{B}, \\
\sigma_{\|} & \approx \frac{n e^{2}}{m_{e} v_{e n}}=\frac{n e}{B \Theta_{0} \sqrt{\psi}}
\end{aligned}
$$

are the total Pedersen, Hall, and parallel conductivities, respectively. Using Eq. (5), we obtain that $\Theta_{0} \sqrt{\psi}=v_{e n} / \Omega_{e} \ll 1$ and $\Theta_{0} / \sqrt{\psi}=\Omega_{i} / v_{i n} \ll 1$, so that at altitudes of interest we have $\sigma_{P} \ll \sigma_{H} \ll \sigma_{\|}$and $\sigma_{P} / \sigma_{||}=\epsilon^{2}$, where $\epsilon$ is defined by Eq. (6).

Further, to adjust for anisotropic conductivities, we rescale the "primed" coordinates as

$x_{1,2}^{\prime}=X_{1,2}^{\prime}, \quad x_{3}^{\prime}=\frac{X_{3}^{\prime}}{\epsilon}$.

Unlike rotation, however, this anisotropic rescaling is not a unitary transformation and it changes the volume element such that $d X_{1}^{\prime} d X_{2}^{\prime} d X_{3}^{\prime}=\epsilon d x_{1}^{\prime} d x_{2}^{\prime} d x_{3}^{\prime}$, so that the plasma density in the new coordinates becomes $N=n / \epsilon$.

We will denote the vector components in the new coordinates by subscripts $X_{i}^{\prime}$. The covariant vector of the electric field transforms according to $E_{X_{i}^{\prime}}=\sum_{k=1}^{3}\left(\partial x_{i}^{\prime} / \partial X_{k}^{\prime}\right) E_{k}^{\prime}$, so that its components in the new coordinates become $E_{X_{1,2}^{\prime}}=E_{1,2}^{\prime}$ and $E_{X_{3}^{\prime}}=E_{3}^{\prime} / \epsilon$.

The current density in the renormalized coordinates $X_{j}^{\prime}$ transforms according to $J_{X_{i}}^{\prime}=(N / n) \sum_{k=1}^{3}\left(\partial X_{i}^{\prime} / \partial x_{k}^{\prime}\right) j_{k}^{\prime}$, so that its components in the new coordinates become

$$
\begin{aligned}
& J_{X_{1}^{\prime}}=K\left(E_{X_{1}^{\prime}}+q E_{X_{2}^{\prime}},\right. \\
& J_{X_{2}^{\prime}}=K\left(-q E_{X_{1}^{\prime}}+E_{X_{2}^{\prime}},\right. \\
& J_{X_{3}^{\prime}}=K E_{X_{3}^{\prime}},
\end{aligned}
$$

where

$$
\begin{aligned}
& K=\frac{\sigma_{\mathrm{P}}}{\epsilon}=\epsilon \sigma_{\|}=\left(\frac{1+\psi}{\psi}\right)^{1 / 2} \frac{e n}{B}, \\
& q=\frac{\sigma_{\mathrm{H}}}{\sigma_{\mathrm{P}}}=\frac{v_{i n}}{(1+\psi) \Omega_{i}}=\frac{\sqrt{\psi}}{(1+\psi) \Theta_{0}} \gg 1 .
\end{aligned}
$$

As a result of this scaling transformation, the quasineutrality equation $\nabla \cdot \boldsymbol{J}=0$ reduces to 3-D Laplace's equation for the electrostatic potential $\Phi$,

$\sum_{i=1}^{3} \frac{\partial^{2} \Phi}{\partial X_{i}^{\prime 2}}=0$. 
Due to the small value of $\epsilon$, Eq. (6), all sizes in the $X_{3}^{\prime}$ direction (i.e., along $\boldsymbol{B}$ ) shrink dramatically. As a result, the ellipsoid determined by Eq. (3) becomes oblate in this direction, while its axes become almost parallel to the coordinate axes $X_{i}$. To make them exactly parallel and restore the simple canonical form of the rescaled ellipsoid, we rotate the coordinate system around the $X_{2}^{\prime}$-axis through a small, but still unknown, angle $\chi$ to the final coordinates $X_{j}$,

$X_{1}^{\prime}=X_{1} \cos \chi-X_{3} \sin \chi$,

$X_{2}^{\prime}=X_{2}$,

$X_{3}^{\prime}=X_{1} \sin \chi+X_{3} \cos \chi$.

The entire transformations from $X_{i}$ to $x_{k}$ and back are related as

$$
\left[\begin{array}{c}
\frac{\partial x_{1}}{\partial X_{1}}, \frac{\partial x_{1}}{\partial X_{3}} \\
\frac{\partial x_{3}}{\partial X_{1}}, \frac{\partial x_{3}}{\partial X_{3}}
\end{array}\right]=\frac{1}{\epsilon}\left[\begin{array}{rr}
\frac{\partial X_{3}}{\partial x_{3}}, & -\frac{\partial X_{1}}{\partial x_{3}} \\
-\frac{\partial X_{3}}{\partial x_{1}}, & \frac{\partial X_{1}}{\partial x_{1}}
\end{array}\right],
$$

$\partial x_{2} / \partial X_{2}=\partial X_{2} / \partial x_{2}=1$, with the Jacobians given by

$\operatorname{det}\left[\frac{\partial\left(X_{i}\right)}{\partial\left(x_{k}\right)}\right]=\left(\operatorname{det}\left[\frac{\partial\left(x_{k}\right)}{\partial\left(X_{i}\right)}\right]\right)^{-1}=\epsilon$.

The explicit expressions for $x_{i}\left(X_{k}\right)$ and $X_{k}\left(x_{i}\right)$ are given by

$$
\begin{aligned}
x_{1} & =\left[\left(1+\epsilon^{-1} \cot \theta \tan \chi\right) X_{1}\right. \\
& \left.+\left(\epsilon^{-1} \cot \theta-\tan \chi\right) X_{3}\right] \sin \theta \cos \chi, \\
x_{3} & =\left[\left(\epsilon^{-1} \tan \chi-\cot \theta\right) X_{1}\right. \\
& \left.+\left(\epsilon^{-1}+\cot \theta \tan \chi\right) X_{3}\right] \sin \theta \cos \chi, \\
X_{1} & =\left[(1+\epsilon \cot \theta \tan \chi) x_{1}\right. \\
& \left.+(\epsilon \tan \chi-\cot \theta) x_{3}\right] \sin \theta \cos \chi, \\
X_{3} & =\left[(\epsilon \cot \theta-\tan \chi) x_{1}\right. \\
& \left.+(\epsilon+\cot \theta \tan \chi) x_{3}\right] \sin \theta \cos \chi .
\end{aligned}
$$

The electric field transforms according to

$$
\begin{aligned}
& {\left[\begin{array}{c}
E_{X_{1}} \\
E_{X_{3}}
\end{array}\right]=\left[\begin{array}{c}
\frac{\partial x_{1}}{\partial X_{1}}, \frac{\partial x_{3}}{\partial X_{1}} \\
\frac{\partial x_{1}}{\partial X_{3}}, \frac{\partial x_{3}}{\partial X_{3}}
\end{array}\right]\left[\begin{array}{c}
E_{1} \\
E_{3}
\end{array}\right],} \\
& E_{X_{2}}=E_{2} \text {, and } \\
& {\left[\begin{array}{c}
E_{1} \\
E_{3}
\end{array}\right]=\left[\begin{array}{l}
\frac{\partial X_{1}}{\partial x_{1}}, \frac{\partial X_{3}}{\partial x_{1}} \\
\frac{\partial X_{1}}{\partial x_{3}}, \frac{\partial X_{3}}{\partial x_{3}}
\end{array}\right]\left[\begin{array}{c}
E_{X_{1}} \\
E_{X_{3}}
\end{array}\right] .}
\end{aligned}
$$

To determine the unknown rotation angle, $\chi$, we require the coordinates $X_{k}$ to be the new ellipsoid axes. Substituting Eq. (A11a) into Eq. (3), equating the factor in front of $X_{1} X_{3}$ to zero, and denoting $\delta \equiv a_{3}^{2} / a_{1}^{2}, C \equiv \cot \theta, \tan \chi \equiv C \epsilon x$, we obtain

$$
(x-1)\left(1+\epsilon^{2} C^{2} x\right)+\left(1+C^{2} x\right)\left(1-\epsilon^{2} x\right) \delta=0 .
$$

To the first-order accuracy with respect to $\delta \ll 1$, the appropriate solution of this quadratic equation $(|x| \sim 1)$ is given by $x \approx 1-\left(1+C^{2}\right) \delta=1-\delta / \sin ^{2} \theta$, so that we obtain

$\tan \chi \approx \epsilon\left(1-\frac{a_{3}^{2}}{a_{1}^{2} \sin ^{2} \theta}\right) \cot \theta$,

and hence, to the first-order accuracy with respect to $\epsilon^{2} \ll 1$,

$\cos \chi \approx 1-\frac{\epsilon^{2} \cot ^{2} \theta}{2}$.

Substituting Eq. (A13) to Eq. (A11b), to the first-order accuracy with respect to small parameters $a_{3}^{2} / a_{1}^{2}$ and $\epsilon^{2}$, we obtain

$$
\begin{aligned}
X_{1} & \approx\left(1+\frac{\epsilon^{2} \cot ^{2} \theta}{2}\right) x_{1} \sin \theta \\
& -\left[1-\epsilon^{2}\left(1+\frac{\cot ^{2} \theta}{2}\right)\right] x_{3} \cos \theta, \\
X_{3} & \approx \frac{\epsilon}{\sin \theta}\left\{\frac{a_{3}^{2}}{a_{1}^{2}} x_{1} \cot \theta+\left[1-\left(\frac{a_{3}^{2}}{a_{1}^{2}}+\frac{\epsilon^{2}}{2}\right) \cot ^{2} \theta\right] x_{3}\right\} .
\end{aligned}
$$

Now we obtain the equation for the potential $\Phi$, using the quasineutrality relation $\nabla \cdot \boldsymbol{J}=0$. From Eq. (A6), using the unitary rotational transformations for the current density,

$$
\begin{aligned}
& J_{X_{1}}=J_{X_{1}}^{\prime} \cos \chi+J_{X_{3}}^{\prime} \sin \chi, \\
& J_{X_{2}}=J_{X_{2}}^{\prime}, \\
& J_{X_{3}}=-J_{X_{1}}^{\prime} \sin \chi+J_{X_{3}}^{\prime} \cos \chi,
\end{aligned}
$$

and the electric field,

$$
\begin{aligned}
& E_{X_{1}}^{\prime}=E_{X_{1}} \cos \chi-E_{X_{3}} \sin \chi, \\
& E_{X_{2}}^{\prime}=E_{X_{2}}, \\
& E_{X_{3}}^{\prime}=E_{X_{1}} \sin \chi+E_{X_{3}} \cos \chi,
\end{aligned}
$$


we obtain to the zeroth-order accuracy

$$
\begin{aligned}
J_{X_{1}} & =K\left(E_{X_{1}}+q E_{X_{2}} \cos \chi\right) \approx K\left(E_{X_{1}}+q E_{X_{2}}\right), \\
J_{X_{2}} & =K\left(-q E_{X_{1}} \cos \chi+E_{X_{2}}+q E_{X_{3}} \sin \chi\right) \\
& \approx K\left[-q E_{X_{1}}+E_{X_{2}}+\left(\frac{\psi}{1+\psi}\right)^{1 / 2} E_{X_{3}} \cot \theta\right], \\
J_{X_{3}} & =K\left(-q E_{X_{2}} \sin \chi+E_{X_{3}}\right) \\
& \approx K\left[-\left(\frac{\psi}{1+\psi}\right)^{1 / 2} E_{X_{2}} \cot \theta+E_{X_{3}}\right] .
\end{aligned}
$$

Here we have used Eq. (A13) along with Eqs. (6) and (A7b). Equation (A15) keeps the same isotropic diagonal terms corresponding to the renormalized combined Pedersen-parallel conductivity as in Eq. (A6). However, the final rotation through the small angle $\chi$ introduces additional anisotropic off-diagonal terms corresponding to the renormalized Hall conductivity. At the constant plasma density, antisymmetric Hall terms do not contribute to the current divergency, so that expressing the field as $E_{X_{i}}=-\partial \Phi / \partial X_{i}$, we obtain from $\partial J_{X_{i}} / \partial X_{i}=0$ Laplace's Eq. (9), equivalent to Eq. (A8).

\section{Appendix B}

\section{Simplification of integrals in Eq. (27)}

In this appendix we simplify the integrals involved in Eq. (27). First, we express them in terms of elementary functions and canonical elliptic integrals of the first and second kind defined here as

$$
\begin{aligned}
& F(z ; k) \equiv \int_{0}^{z} \frac{d t}{\sqrt{\left(1-t^{2}\right)\left(1-k^{2} t^{2}\right)}} \\
& E(z ; k) \equiv \int_{0}^{z} \sqrt{\frac{1-k^{2} t^{2}}{1-t^{2}}} d t
\end{aligned}
$$

(e.g., Abramowitz and Stegun, 1970). Then, employing the inequalities between the ellipsoid semi-axes $A_{i}$, Eq. (12), we approximate these integrals with elementary functions. Finally, we calculate the normalization constants $\Lambda_{i}$ of Eq. (27b).

For each $i=1,2,3$, the integrals in Eqs. (27) have similar forms but their explicit expressions in terms of the canonical elliptic integrals differ significantly. All elliptic integrals, however, have the same arguments $z$ and $k$ :

$$
z(\xi)=\left(\frac{\xi+A_{3}^{2}}{\xi+A_{2}^{2}}\right)^{1 / 2}, \quad k=\left(\frac{A_{1}^{2}-A_{2}^{2}}{A_{1}^{2}-A_{3}^{2}}\right)^{1 / 2},
$$

where $z(\xi)$ varies between $A_{3} / A_{2}$ and 1 . The explicit expressions of the integrals are given by

$$
\begin{aligned}
& \int_{0}^{\xi} \frac{d \xi}{\left(A_{1}^{2}+\xi\right) R_{\xi}}=\frac{2(Q(\xi)-Q(0))}{\left(A_{1}^{2}-A_{3}^{2}\right)^{1 / 2}\left(A_{1}^{2}-A_{2}^{2}\right)} \\
& +\frac{2}{A_{1}^{2}-A_{3}^{2}}\left\{\left[\frac{\xi+A_{3}^{2}}{\left(\xi+A_{1}^{2}\right)\left(\xi+A_{2}^{2}\right)}\right]^{1 / 2}-\frac{A_{3}}{A_{1} A_{2}}\right\}
\end{aligned}
$$

$$
\int_{0}^{\xi} \frac{d \xi}{\left(A_{2}^{2}+\xi\right) R_{\xi}}=\frac{2(P(\xi)-P(0))}{\left(A_{1}^{2}-A_{3}^{2}\right)^{1 / 2}\left(A_{2}^{2}-A_{3}^{2}\right)\left(A_{1}^{2}-A_{2}^{2}\right)},
$$

$$
\int_{0}^{\xi} \frac{d \xi}{\left(A_{3}^{2}+\xi\right) R_{\xi}}=\frac{2(S(0)-S(\xi))}{\left(A_{1}^{2}-A_{3}^{2}\right)^{1 / 2}\left(A_{2}^{2}-A_{3}^{2}\right)},
$$

where

$$
\begin{aligned}
Q(\xi) & =F(z(\xi) ; k)-E(z(\xi) ; k), \\
P(\xi) & =\left(A_{3}^{2}-A_{2}^{2}\right) F(z(\xi) ; k)+\left(A_{1}^{2}-A_{3}^{2}\right) E(z(\xi) ; k), \\
S(\xi) & =E(z(\xi) ; k) \\
& +\left(A_{2}^{2}-A_{3}^{2}\right)\left[\frac{A_{1}^{2}+\xi}{\left(A_{1}^{2}-A_{3}^{2}\right)\left(A_{3}^{2}+\xi\right)\left(A_{2}^{2}+\xi\right)}\right]^{1 / 2} .
\end{aligned}
$$

These exact expressions are valid for arbitrary $A_{j}$ ordered according to $A_{1}>A_{2}>A_{3}$.

Now, we approximate the elliptic integrals by elementary functions. Under conditions of $A_{1} \gg A_{2,3}$, see Eq. (12), the parameter $k$ is close to unity, $\Delta \equiv 1-k^{2} \ll 1$. Then in the most of the entire $z$-range, $1-z \gtrsim \Delta$, we have

$$
\begin{aligned}
& F(z ; k) \approx \frac{5-k^{2}}{8} \ln \left(\frac{1+z}{1-z}\right)-\frac{\left(1-k^{2}\right) z}{4\left(1-z^{2}\right)}, \\
& E(z ; k) \approx z-\frac{1-k^{2}}{2}\left[z-\frac{1}{2} \ln \left(\frac{1+z}{1-z}\right)\right] .
\end{aligned}
$$

This approximation fails in a small vicinity of the top boundary, $1-z \ll \Delta$. For all $1-z \ll 1$, we have

$$
\begin{aligned}
& F(z ; k) \approx\left(\frac{5-k^{2}}{4}\right) \ln \left[\frac{4}{\sqrt{2(1-z)}+\sqrt{3-k^{2}-2 z}}\right] \\
&+\frac{z-k^{2}}{4} \sqrt{\frac{2(1-z)}{3-k^{2}-2 z}}-\frac{1-k^{2}}{4}, \\
& E(z ; k) \approx \frac{3+k^{2}}{4}-\sqrt{\frac{(1-z)\left(3-k^{2}-2 z\right)}{2}} \\
&-\frac{1-k^{2}}{2} \ln \left(\sqrt{\frac{1-z}{8}}+\sqrt{\frac{3-k^{2}-2 z}{16}}\right) .
\end{aligned}
$$


Equations (B5) and (B6) match at an intermediate region $1-z \sim \Delta$ and they approximate the exact elliptic integrals with a remarkable accuracy. For example, for $A_{1,2,3}$ which are proportional to 10,1 , and 0.1 respectively $(k=10 / \sqrt{101}$, $\Delta \approx 0.01)$, the relative mismatch between the exact and proper approximate values of the elliptic integrals is $\sim 10^{-4}$ in the matching region and is much less beyond it. Even for $A_{1,2,3}$ as close to each other as 5,4 , and $3(k=3 / 4, \Delta \approx 0.44)$, the relative mismatch between the exact elliptic integrals and their proper approximate values is only a few percent at most.

Equations (B4) to (B6) accurately approximate the integrals in Eq. (B3) over the entire space: Eq. (B5) covers $\xi \lesssim A_{1}^{2}$, while Eq. (B6) covers $\xi \gg A_{2}^{2}$. Because $A_{1} \gg A_{2}$ the two regions overlap.

Now, we find simplified approximate expressions for the normalization constants

$\Lambda_{i}=\left(A_{i}^{3} / 2\right) \int_{0}^{\infty} d \xi /\left[\left(A_{i}^{2}+\xi\right) R_{\xi}\right]$, Eq. (27b). For these integrals, Eq. (B4) requires $z(0)=A_{3} / A_{2} \ll 1$ and $z(\infty)=1$. For the former, we use Eq. (B5), while for the latter we use Eq. (B6). Expanding all resultant expressions in powers of the small ratios $A_{2} / A_{1}$ and $A_{3} / A_{2}$ (up to the second-order accuracy), we obtain from Eq. (B3)

$$
\begin{aligned}
& \Lambda_{1} \approx \ln \frac{4 A_{1}}{A_{2}}-1-\frac{A_{3}}{A_{2}}+\left(\frac{3}{4} \ln \frac{4 A_{1}}{A_{2}}-1\right) \frac{A_{2}^{2}}{A_{1}^{2}}+\frac{A_{3}^{2}}{2 A_{2}^{2}}, \\
& \Lambda_{2} \approx \frac{A_{2}}{A_{1}}\left[1-\frac{A_{3}}{A_{2}}+\left(\frac{3}{4}-\frac{1}{2} \ln \frac{4 A_{1}}{A_{2}}\right) \frac{A_{2}^{2}}{A_{1}^{2}}+\frac{A_{3}^{2}}{A_{2}^{2}}\right], \\
& \Lambda_{3} \approx \frac{A_{3}^{2}}{A_{1} A_{2}}\left(1-\frac{A_{3}}{A_{2}}-\frac{A_{3} A_{2}}{2 A_{1}^{2}} \ln \frac{4 A_{1}}{A_{2}}+\frac{A_{3}^{2}}{A_{2}^{2}}\right) .
\end{aligned}
$$

These expressions provide accurate approximations of $\Lambda_{j}$ for $A_{2} / A_{1}, A_{3} / A_{2}<0.2$ by elementary functions. The relative mismatch between the exact integrals and approximate values is below one percent. We use Eq. (B7) in Section 3.2.

Acknowledgements. This work was supported by National Science Foundation Ionospheric Physics Grants No. ATM-0332354, ATM0334906, and ATM-0334256.

Topical Editor M. Pinnock thanks W. G. Elford and another anonymous referee for their help in evaluating this paper.

\section{References}

Abramowitz, M. and Stegun, I.: Handbook of Mathematical Functions, Dover Publications, INC., ninth edn., 1970.

Baggaley, W. J. and Webb, T. H.: The Geomagnetic Control of the diffusion of meteoric ionozation, Planet. Space Sci., 28, 9971001, 1980.

Bronshten, V. A.: Physics of Meteoric Phenomena, Reidel Publishing Company, Dordrecht-Boston-Lancaster, 1983.

Ceplecha, Z., Borovicka, J., Elford, W. G., Revelle, D. O., Hawkes, R. L., Porubcan, V., and Simek, M.: Meteor Phenomena and Bodies, Space Sci. Rev., 84, 327-471, 1998.
Chapin, E.: Simultaneous observations of meteors and the equatorial electrojet at Jicamarca, Ph.D. thesis, Univ. Illinois, Urbana Chamaign, Illinois, 1996.

Chapin, E. and Kudeki, E.: Plasma-wave excitation on meteor trails in the equatorial electrojet, Geophys. Res. Lett., 21, 2433-2436, 1994a.

Chapin, E. and Kudeki, E.: Radar interferometric imaging studies of long duration meteor echo observed at Jicamarca, J. Geophys. Res., 99, 8937-8949, 1994b.

Dimant, Y. S. and Milikh, G. M.: Model of anomalous electron heating in the E region: 1. Basic theory, J. Geophys. Res., 108, 1350, doi:10.1029/2002JA009524, 2003.

Dimant, Y. S. and Oppenheim, M. M.: Ion thermal effects on E-region instabilities: linear theory, J. Atmos. Terr. Phys., 66, 1639-1654, 2004.

Dimant, Y. S. and Oppenheim, M. M.: Meteor trail diffusion and fields: 1. Simulations, J. Geophys. Res., 111, A12312, doi:10. 1029/2006JA011797, 2006a.

Dimant, Y. S. and Oppenheim, M. M.: Meteor trail diffusion and fields: 2. Analytical theory, J. Geophys. Res., 111, A12313, doi: 10.1029/2006JA011798, 2006b.

Dyrud, L. P., Oppenheim, M. M., Close, S., and Hunt, S.: Interpretation of non-specular radar meteor trails, Geophys. Res. Lett., 29, 2012, doi:10.1029/2002GL015953, 2002.

Dyrud, L. P., Denney, K., Urbina, J., Janches, D., Kudeki, E., and Franke, S.: The Meteor Flux: it Depends how you Look, Earth Moon and Planets, 95, 89-100, doi:10.1007/ s11038-005-9001-6, 2004.

Elford, W. G. and Elford, M. T.: The effective diffusion coefficient of meteor trails above $100 \mathrm{~km}$, in: ESA SP-495: Meteoroids 2001 Conference, pp. 357-359, 2001.

Galligan, D. P., Thomas, G. E., and Baggaley, W. J.: On the relationship between meteor height and ambipolar diffusion, J. Atmos. Terr. Phys., 66, 899-906, doi:10.1016/j.jastp.2004.03.002, 2004.

Gurevich, A. V.: Nonlinear phenomena in the ionosphere, SpringerVerlag, New York, 1978.

Gurevich, A. V., Borisov, N. D., and Milikh, G. M.: Physics of microwave discharges: artificially ionized regions in the atmosphere, Gordon and Breach, UK, 208 pp., 1997.

Hocking, W. K.: Experimental Radar Studies of Anisotropic Diffusion of High Altitude Meteor Trails, Earth, Moon, and Planets, 95, 671-679, 2004.

Hysell, D. L. and Drexler, J.: Polarization of elliptic E region plasma irregularities and implications for coherent radar backscatter from Farley-Buneman waves, Radio Sci., 41, RS4015, doi:10.1029/2005RS003424, 2006.

Jones, J. and Jones, W.: Oblique-scatter of radio waves from meteor trains: Full-wave calculations, Planet. Space Sci., 39, 1289 1296, doi:10.1016/0032-0633(91)90043-A, 1991.

Jones, W. and Jones, J.: Ionic diffusion in meteor trains, J. Atmos. Terr. Phys., 52, 185, 1990.

Kaiser, T. R. and Closs, T.: Polarization of elliptic E region plasma irregularities and implications for coherent radar backscatter from Farley-Buneman waves, Phil. Mag., 43, 1-29, 1952.

Kelley, M. C.: The Earth's Ionosphere, Academic, San Diego, California, 1989.

Landau, L. D. and Lifshitz, E. M.: Electrodynamics of Continuous Media, Eddison-Wesley, Reading, MA, 1960.

Lebedinec, V. N. and Sosnova, A. K.: Radio reflections from meteor 
trails, in: Physics and Dynamics of Meteors, edited by: Kresak, L. and Millman, P. M., pp. 27-44, Reidel, Dordrecht, 1968.

Levitskii, S. M., Abdrakhmanov, N., and Timchenko, V. P.: The effect of the geomagnetic field on the diffusion of meteor trails, Radiophys. Quantum Electr., 25, 1240-1243, 1982.

McEwan, M. J. and Phillips, L. F.: Chemistry of the atmosphere, New York, Halsted Press, 309 p., 1975.

Milikh, G. M. and Dimant, Y. S.: Model of anomalous electron heating in the E region: 2. Detailed numerical modeling, J. Geophys. Res., 108, 1351, doi:10.1029/2002JA009527, 2003.

Oppenheim, M. M. and Dimant, Y.: Meteor induced ridge and trough formation and the structuring of the nighttime E-region ionosphere, Geophys. Res. Lett., 33, L24105, doi:10.1029/ 2006GL028267, 2006.
Oppenheim, M. M., vom Endt, A. F., and Dyrud, L. P.: Electrodynamics of meteor trail evolution in the equatorial E-region ionosphere, Geophys. Res. Lett., 27, 3173-3176, 2000.

Papadopoulos, K., Milikh, G., Gurevich, A., Drobot, A., and Shanny, R.: Ionization rates for atmospheric and ionospheric breakdown, J. Geophys. Res., 98, 17593-17596, doi:10.1029/ 93JA00795, 1993.

Poulter, E. M. and Baggaley, W. J.: Radiowave scattering from meteoric ionization., J. Atmos. Terr. Phys., 39, 757-768, 1977.

Schunk, R. W. and Nagy, A. F.: Ionospheres, Cambridge University Press, 2000.

Tsang, K., Papadopoulos, K., Drobot, A., Vitello, P., Wallace, T., and Shanny, R.: RF ionization of the lower ionosphere, Radio Sci., 26, 1345-1360, 1991. 